\title{
A serendipitous survey for variability amongst the massive stellar population of Westerlund $1^{\star, \star \star}$
}

\author{
J. S. Clark ${ }^{1}$, B. W. Ritchie ${ }^{1,2}$, and I. Negueruela ${ }^{3}$ \\ 1 Department of Physics and Astronomy, The Open University, Walton Hall, Milton Keynes MK7 6AA, UK \\ e-mail: jsc@star.ucl.ac.uk \\ 2 IBM United Kingdom Laboratories, Hursley Park, Winchester, Hampshire S021 2JN, UK \\ 3 Departamento de Física, Ingeniería de Sistemas y Teoría de la Señal, Universidad de Alicante, Apdo. 99, 03080 Alicante, Spain
}

Received 7 December 2009 / Accepted 17 February 2010

\section{ABSTRACT}

\begin{abstract}
Aims. Massive stars are known to demonstrate significant spectroscopic and photometric variability over a wide range of timescales. However the physical mechanisms driving this behaviour remain poorly understood. Westerlund 1 presents an ideal laboratory for studying these processes in a rich, coeval population of post-main sequence stars and we present a pathfinding study aimed at characterising their variability.

Methods. To do this we utilised the large body of spectroscopic and photometric data that has accumulated for Wd1 during the past decade of intensive studies, supplemented with the sparser historical observations extending back to the early 1960s.

Results. Despite the heterogeneous nature of this dataset, we were able to identify both spectroscopic and photometric variability amongst every class of evolved massive star present within Wd 1. Spectroscopic variability attributable to both wind asphericity and photospheric pulsations was present amongst both the hot and cool hypergiants and the former, also with the Wolf Rayets. Given the limitations imposed by the data, we were unable to determine the physical origin of the wind structure inferred for the OB supergiants, noting that it was present in both single pulsating and binary stars. In contrast we suspect that the inhomogineities in the winds of the Wolf Rayets are driven by binary interactions and, conversely, by pulsations in at least one of the cool hypergiants. Photospheric pulsations were found for stars ranging from spectral types as early as $\mathrm{O} 9 \mathrm{I}$ through to the mid $\mathrm{F} \mathrm{Ia}^{+}$yellow hypergiants - with a possible dependence on the luminosity class amongst the hot supergiants. The spectroscopically variable red supergiants (M2-5 Ia) are also potential pulsators but require further observations to confirm this hypothesis. Given these findings it was therefore rather surprising that, with the exception of W243, no evidence of the characteristic excursions of both luminous blue variables and yellow hypergiants was found. Nevertheless, future determination of the amplitude and periodicity of these pulsations as a function of temperature, luminosity and evolutionary state holds out the tantalising possibility of constraining the nature of the physical mechanisms driving the instabilities that constrain and define stellar evolution in the upper reaches of the HR diagram. Relating to this, the lack of secular evolution amongst the cool hypergiants and the presence of both high-luminosity yellow hypergiants and red supergiants within Wd1 potentially place strong constraints on post-main sequence evolutionary pathways, with the latter result apparently contradicting current theoretical predictions for $>25 M_{\odot}$ stars at solar metallicites.
\end{abstract}

Key words. stars: evolution - stars: variables: general - supergiants

\section{Introduction}

A large body of observational evidence exists that massive postmain sequence (MS) stars are subject to a variety of (pulsational) instabilities (e.g. Humphreys \& Davidson 1994; Sterken 1977; Burki 1978; de Jager 1998; Wood et al. 1983). Indeed, it has been supposed that such instabilities contribute to the absence of cool $(T<10 \mathrm{kK})$ hypergiants above $\log \left(L / L_{\odot}\right) \sim 5.8$, giving rise to the the Humphreys Davidson limit that spans the upper reaches of the HR diagram.

Characterisation of such instabilities is important for two reasons. Firstly, one might hope to use the pulsational properties to investigate the internal structure of massive stars via asteroseismology. Secondly, there is increasing reason to suppose that

* Based on observations collected at the European Southern Observatory, Paranal and La Silla Observatories under programme IDs ESO 067.D-0211, 069.D-0039, 071.D-0151, 073.D-0327, 075.D-0388, 081.D-0324 and 383.D-0633.

$\star \star$ Tables 2 and 4 are only available in electronic form at http://www. aanda.org
MS mass loss is insufficient to permit the transition of high-mass stars from unevolved O-type objects to H-depleted Wolf Rayet stars, and instead that the H-rich mantle is shed in short-lived impulsive events associated with the "zoo" of transitional objects. Indeed observational evidence of this hypothesis is provided by the presence of fossil ejecta surrounding luminous blue variables (LBVs: e.g. Clark et al. 2003), yellow hypergiants (YHGs; Castro-Carrizo et al. 2007) and red supergiants (RSGs; Schuster et al. 2006) and the direct association of dramatic increases in mass loss with episodes of instability in both hot and cool luminous stars ( $\eta$ Car and $\rho$ Cas, respectively; Smith \& Gehrz 1998; Lobel et al. 2003). Thus, determining the duration, duty cycle and mass loss rate of such events appears to be necessary for the construction of a theory of massive stellar evolution.

These instabilities are manifest in both photometric and spectroscopic variability, but other physical processes, such as large and small scale wind inhomogineities, also lead to similar behaviour. Line driven winds are intrinsically unstable, leading to stochastic clumping (e.g. Owocki 2000), while hydrodynamical simulatons by Cranmer \& Owocki (1996) demonstrate 
that large-scale photospheric perturbations - originating in nonradial pulsations and/or magnetic fields - can lead to large scale rotating wind structures. Moreover, spectrophotometric variability is an important observational signature of wind collision zones in massive binaries (e.g. Lewis et al. 1993; Stevens \& Howarth 1999). Of these phenomena, the degree of wind clumping present in massive stellar winds is critical to the observational determination of the mass loss rate, while the characterisation of the properties of the massive binary population is important for fields as diverse as star formation, the nature of $\mathrm{SNe}$ progenitors and the formation channels of X-ray binaries.

Consequently, considerable observational effort has been expended in the identification and characterisation of variability in massive (binary) stars. Such studies have typically centred on field stars, with the attendant difficulties of the determination of stellar luminosity and hence mass and age. However the development of multiplexing spectrographs and the identification of a large number of massive young Galactic clusters - of which Westerlund 1 is an examplar (henceforth $\mathrm{Wd} 1$ ) - presents a unique opportunity to advance this field, offering a large coeval population of massive stars of identical initial metallicity and well constrained masses.

First identified by Westerlund (1961), Wd1 was subject to sporadic optical and near-IR photometric studies in the following four decades (Sect. 2), with the high reddening towards it preventing a spectroscopic study until that of Westerlund (1987; West87). However, despite the discovery of a remarkable population of both early and late super/hypergiants, the cluster languished in relative obscurity until the turn of the century, when modern spectroscopic studies - motivated by the unexpected radio properties of $\mathrm{Wd} 1$ - first identified a rich population of Wolf-Rayets (WRs; Clark \& Negueruela 2002) and subsequently revealed $\mathrm{Wd} 1$ to be the first example of a super star cluster identified in the Galaxy (Clark et al. 2005a; henceforth C05). In the following decade, $\mathrm{Wd} 1$ has been the subject of a large number of studies from X-ray to radio wavelengths aimed at determining both bulk cluster properties and those of the individual constituent stars. The combined dataset therefore offers the potential of investigating the variability of cluster members over a significant time period ( $\geq 40 \mathrm{yr}$ for the brightest stars; Sect. 2) and we present the results of such an analysis in this manuscript. In Sect. 2 we present and describe the reduction of the new data included in this work and discuss the utilisation of data available in previous studies. In Sects. 3-7 we analyse the available data as a function of evolutionary state and provide a discussion and summary in Sect. 8.

\section{Observations and data reduction}

Between its discovery in 1961 and the era of intensive observations post 2001, Wd 1 was the subject of a number of photometric and spectroscopic studies ranging from optical to mid-IR wavelengths; these are summarised in Table 1. Unfortunately, in many cases observations collected in single works were not contemporaneous, complicating analysis; an important example is the photometry and spectroscopy presented by West87, which were obtained 15 years apart. An additional difficulty in comparing multiepoch photometry is the different filter and detector (photographic plate, photometer and CCD) combinations employed by the various studies, for which accurate spectral responses were unavailable. Combined with different extraction techniques (important due to the crowded nature of the cluster) and the lack of appropiate red standards, we found the number of observationally robust results that could be drawn from the
Table 1. Summary of published observations utilised in this study.

\begin{tabular}{lll}
\hline \hline Year & Dataset & Reference \\
\hline 1961 & $V I$ band phot. & Borgman et al. (1970) \\
1966 & VRI band phot. & West87 \\
1969 & $K$ band phot. & Borgman et al. (1970) \\
1973 & $V$ +narrow band phot. & Lockwood (1974) \\
$1973-4$ & $2-20 \mu$ m phot. & Koornneef (1977) \\
1995 & $V I$ band phot. & Piatti et al. (1998) \\
2001 & $B V R I$ band phot. & C05 \\
2006 & $B V R I$ band phot. & Bonanos (2007) \\
\hline 1981 & $R I$ band spec. & West87 \\
2001 & $R I$ band spec. & C05 \\
2002 & $R I$ band spec. & C05 \\
2003 & $R I$ band spec. & Negueruela \\
& & \& Clark (2005) \\
2005 & $I J \& H K$ spec. & Crowther et al. (2006) \\
2006 & $K$ band spec. & Mengel \& \\
& & Tacconi-Garman (2007) \\
\hline
\end{tabular}

historical data were limited. Finally, the recent near-IR photometric observations of Brandner et al. (2008) provide no extra information due to the saturation of the near-IR bright supergiants.

\subsection{Photometric data}

Comparative analysis of multiepoch photometric data obtained prior to 2001 was not attempted for the above reasons and, with the exception of West87, the limited number of stars observed in such works, which precluded the determination of empirical offsets between different datasets (e.g. Fig. 1) ${ }^{1}$. Hence the only robust results to be drawn from these observations are the coarse spectral classifications derived from colour indices for the (contemporaneous) datasets presented by West87, Lockwood (1974), Koornneef (1977) and Piatti et al. (1998). Unfortunately these just permit the classification of stars into $\mathrm{OB}$ and $\mathrm{M}$ supergiant classifications, for which we found the former to contain stars of spectral type from late $\mathrm{O}$ to mid $\mathrm{GIa}^{+}$. These results are summarised in Table 2.

These problems persist for modern, post-2001 datasets. This is apparent in the systematic offset between the $V$ band datasets present in C05 and Bonanos (2007; Fig. 1) due to the difficulty in determining the zero point offset because of the lack of appropiate red standards ${ }^{2}$. However the large number of stars in common between West87, C05 and Bonanos (2007) enabled the offsets to be determined empirically and hence a direct comparison to be made between the three epochs (Fig. 1).

This approach allows potentially variable outliers to be identified, albeit with three corrolaries. Firstly observations of a particular star are not always present in each dataset; for example W4, 20, 26, 32, 42, 75 \& 243 are absent from C05. Secondly, observations of individual objects are likewise not present in all 3 wavebands of a given dataset. For example, comparable photometry for W243 between West 87 and Bonanos (2007) is only available in the $V$ band, thus the apparent discrepancy between

\footnotetext{
1 Note that the limited stellar identifications and lack of co-ordinates presented by Piatti et al. (1998) limit the utility of those observations.

2 Bonanos (2007) successfully made such a correction for $R$ and $I$ band, but the methodology employed - comparison of the brightest 100 stars in common between the 2 datasets - was not completely successful for the $V$ band. We suspect this to be because the brightest stars in the $V$ band were dominated by foreground stars of relatively low reddening in comparison to the stars within Wd1.
} 

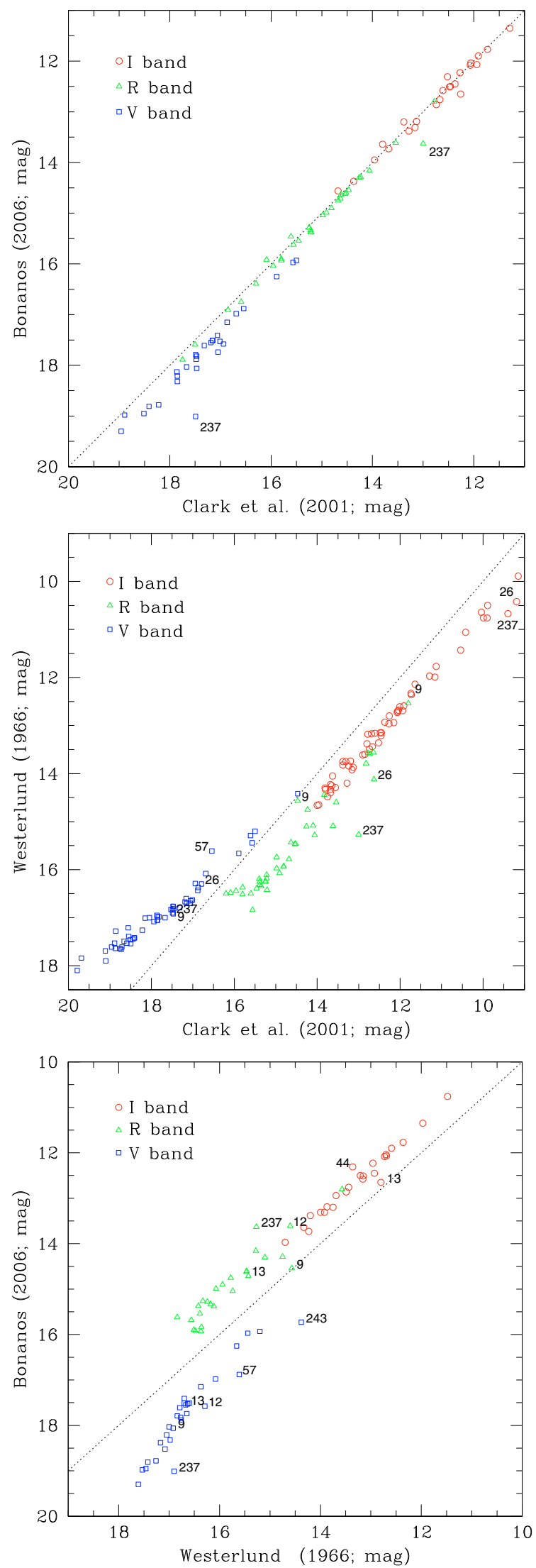

Fig. 1. Comparison of the photometric datasets presented by $\mathrm{C} 05$, Bonanos (2007) and West87. Note the zero point offsets present between all three datasets. In all three panels outliers are flagged, along with observations of such stars in the other wavebands, where available. Also note that, for a given star, photometry is not always available for all three datsets (Sect. 2.1). the two epochs is impossible to verify ${ }^{3}$ (complicated by the fact that the empirical offset is ill defined for stars of this magnitude). Finally, issues arising from filter and photographic emulsion response to very red objects (West87, Bessell 1986) cast doubt upon the reality of the offsets between the data presented by West87 for the RSGs W26 \& 237 and other epochs, although this would not explain the apparent variability of W237 in $V$ \& $R$ bands between 2001-6 which we consider likely to be of astrophysical origin (Sect. 7).

Given the above, in addition to W237 we may only tentatively identify a handful of stars - W9, 57a \& 243 - as potentially variable, although with only 3 epochs of observations over a $20 \mathrm{yr}$ baseline and a lower limit on detectable variations of $>0.5$ mag (based on the scatter between datasets evident in Fig. 1), these are hardly stringent constraints. Nevertheless, foreshadowing the detailed results and discussion presented in Sects. 3-7, both hot and cool transitional stars may demonstrate long term (>decades) variability of many magnitudes amplitude; we would have expected to identify such behaviour photometrically if present within the evolved stellar population of $\mathrm{Wd} 1$.

\subsection{Spectroscopic data}

Prior to the discovery of a rich population of WRs within $\mathrm{Wd1}$, the only spectroscopic survey to have been undertaken was that of West87. Motivated by this discovery, several optical and near-IR studies have subsequently been made (Table 1); the published results of all these works are summarised in Table 2. Given the varied science goals of the proposals from which these observations have been drawn, the resulting spectra sample a number of different wavelength ranges and corresponding resolutions; these are summarised in Table 3 with, where available, a reference for a more detailed description including the reduction procedure adopted. Of these data, those obtained in 2003 June (NTT/EMMI), 2005 March, May \& July (VLT/FLAMES), 2006 February (NTT/EMMI) and 2008 September \& 2009 May \& August (VLT/FLAMES) are published here for the first time, as are the observations of the YHGs from 2004 June (VLT/FORS) with the corresponding data for the OB stars found in Negueruela et al. (2010). In total this amounts to in excess of a hundred new spectra.

Special mention must be made of the optical spectra obtained in 2001 June (C05) and the near-IR spectra obtained in 2006 March (Mengel \& Tacconi-Garman 2007, 2008). The former are of significantly lower resolution and $\mathrm{S} / \mathrm{N}$ than later epochs. Following the analysis presented in Negueruela et al. (2010) we may utilise these data to adequately distinguish between extreme B hypergiants/WNL stars (Group 1 stars following C05, with the additional criteria that $\mathrm{H} \alpha$ must be in emission), late $\mathrm{O} /$ early $\mathrm{B}$ supergiants (Group 2; O9-B2 I), mid-late B super-/hypergiants (Group 3; B2.5-9I ${ }^{(+)}$) and YHGs. Thus, while these data are inadequate for the analysis of line profile variability ${ }^{4}$ they do permit the gross spectral classification of cluster members, hence extending the baseline for the identification of e.g. the characteristic temperature excursions of LBVs by a further year.

The latter spectra sample a relatively poorly studied region of the near-IR waveband, for which a comprehensive spectral atlas has yet to be constructed. Nevertheless, the relative strength

\footnotetext{
3 The same is the case for W44 \& 57a, and W57a in the comparison between West 87 and $\mathrm{C} 05$ datasets.

${ }^{4}$ However they are sufficient to identify dramatic changes in line profiles such as the appearance of $\mathrm{H} \alpha$ emission in W23a and W43a; C05 and Sect. 3.
} 
A\&A 514, A87 (2010)

Table 3. Summary of the properties of the spectroscopic datasets employed in this study.

\begin{tabular}{|c|c|c|c|c|c|c|c|}
\hline Date & MJD & Telescope & Instrument & Setting & $\begin{array}{c}\lambda \\
(\AA)\end{array}$ & Resln. & Reference \\
\hline $23-25 / 06 / 01$ & $52083-5$ & ESO $1.52 \mathrm{~m}$ & Boller \& Chivens & Grat. \#1 & $6000-11000$ & 500 & $\mathrm{C} 05$ \\
\hline $\begin{array}{l}07 / 06 / 02+ \\
06-08 / 06 / 03\end{array}$ & $\begin{array}{c}52432 \\
52796-8\end{array}$ & NTT & EMMI & $\begin{array}{l}\text { Grat. \#7 } \\
\text { Grat.\#6 }\end{array}$ & $\begin{array}{l}6310-7835 \\
8225-8900\end{array}$ & $\begin{array}{l}2600 \\
5000\end{array}$ & $\begin{array}{l}\text { C05 } \\
\text { Negueruela \& Clark (2005) }\end{array}$ \\
\hline $12-13 / 06 / 04$ & $53168-69$ & VLT & $\begin{array}{l}\text { FORS longslit } \\
\text { FORS MXU } \\
\text { FORS MXU }\end{array}$ & $\begin{array}{l}\text { G1028z } \\
\text { G1200R } \\
\text { G1200R } \\
\text { G1028z }\end{array}$ & $\begin{array}{r}7730-9480 \\
5750-7310 \\
7730-9480^{a} \\
5750-7310^{a}\end{array}$ & $\begin{array}{l}\sim 7000 \\
\sim 7000 \\
2560 \\
2140\end{array}$ & Negueruela et al. (2010) \\
\hline $\begin{array}{l}25 / 03 / 05+ \\
29 / 05 / 05+ \\
13 / 07 / 05\end{array}$ & $\begin{array}{l}53454 \\
53519 \\
53564\end{array}$ & VLT & $\begin{array}{l}\text { FLAMES+ } \\
\text { MEDUSA }\end{array}$ & $\begin{array}{l}\text { LR6 } \\
\text { LR8 }\end{array}$ & $\begin{array}{l}6438-7184 \\
8206-9400\end{array}$ & $\begin{array}{l}8600 \\
6500\end{array}$ & Ritchie et al. (2009b) \\
\hline $29-30 / 06 / 05$ & $53550-1$ & NTT & SOFI & $\begin{array}{l}\text { IJ Grism } \\
\text { HK Grism }\end{array}$ & $\begin{array}{c}9500-13500 \\
15000-22700\end{array}$ & $\begin{array}{l}1000 \\
1000\end{array}$ & Crowther et al. (2006) \\
\hline $17 / 02 / 06$ & 53783 & NTT & EMMI & Grat. \#6 & $5740-8730$ & 1500 & Marco \& Negueruela (2009) \\
\hline $11-12 / 03 / 06$ & $53805-6$ & VLT & ISAAC & SWS(MR) & $22490-23730$ & 9000 & $\begin{array}{l}\text { Mengel \& } \\
\text { Tacconi-Garman (2007) }\end{array}$ \\
\hline $\begin{array}{l}20+29 / 06 / 08+ \\
18+24 / 07 / 08+ \\
14+17 / 08 / 08+ \\
04+15 / 09 / 08+ \\
19+25 / 09 / 08+ \\
14+18 / 05 / 09+ \\
20 / 08 / 09\end{array}$ & $\begin{array}{c}54367+76 \\
54665+71 \\
54692+95 \\
54713+24 \\
54728+34 \\
54965+69 \\
55063\end{array}$ & VLT & $\begin{array}{l}\text { FLAMES+ } \\
\text { MEDUSA }\end{array}$ & HR21 & 8484-9001 & 16200 & Ritchie et al. (2009a) \\
\hline
\end{tabular}

Notes. ${ }^{a}$ Note that the nominal wavelength range is given for the 2004 FORS MXU observations; in practice this can be displaced by a few hundred $\AA$ depending on the position of an individual star on the CCD.

of the CO bandheads may permit classification of cool supergiants (Figer et al. 2006; Yamamuro et al. 2007). Mengel \& Tacconi-Garman $(2007,2008)$ use this diagnostic to classify the 4 RSGs within Wd1, while the lack of $\mathrm{CO}$ bandhead absorption features in the spectra of W4, 8a, 9, 12, 32 \& 265 suggest a spectral type of G5 Ia or earlier.

Summaries of the data available for late O/early B supergiants and the more evolved, transitional stars are presented in Tables 4 and 5 respectively. A comparable table for the WRs may be found in Crowther et al. (2006), noting that the three WRs included in Table 5 are the sole objects for which multiple epochs of high resolution data are available, thus permitting analysis of line profile evolution. Given the recent comprehensive analysis by Ritchie et al. (2009b), the LBV W243 has been omitted from this study.

\section{The 09-B2 supergiants}

Prior to 2008, comparatively few spectroscopic data were available for individual O9-B2 supergiants (Table 4), restricting analysis primarily to the detection of line profile variability (LPV) in the $\mathrm{H} \alpha$ line. Of the stars with multiple spectra covering this transition, 12 have two or more observations for which $R \geq 2600$, while a further 6 have two epochs of data of which one is of low resolution $(R \sim 500)$ and $S / N$. Of these 18, 9 stars demonstrated significant $\mathrm{H} \alpha$ LPV. This is illustated for W2a, 6a and 61a in Figs. $2-3^{5}$, while it is apparent for W19, 23a, 28, 30a and 43a by

\footnotetext{
${ }^{5}$ Note that throughout the paper the rest wavelengths of transitions are indicated in figures, while the stellar spectra demonstrate a systemic blueshift of $\sim 40 \mathrm{~km} \mathrm{~s}^{-1}$.
}
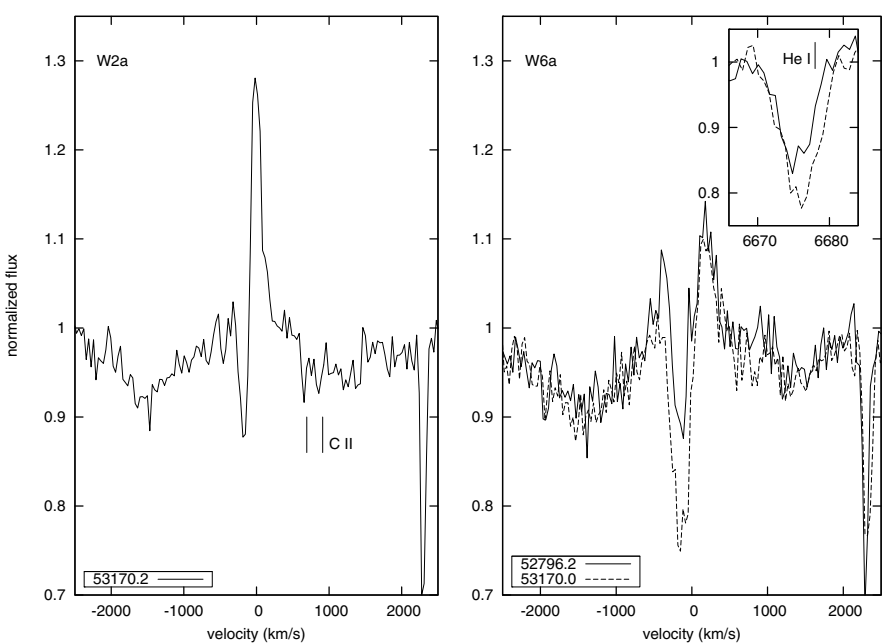

Fig. 2. Left panel: $\mathrm{H} \alpha$ for $\mathrm{W} 2 \mathrm{a}$ (B2 Ia) from 2004 - the line is strongly in emission in this spectrum but was absent in the low $\mathrm{S} / \mathrm{N}$ spectrum from 2001 (not shown). Right panel: $\mathrm{H} \alpha$ profile for W6a (B0.5 Iab) in 2003-4; note the temperature dependent He I $\lambda \lambda 6678,7065 \AA$ lines also appear to vary in strength.

comparison of the spectra presented in C05, Clark et al. (2008) and Negueruela et al. (2010); the spectra of the final example - W28 - are omitted due to their comparatively low $S / N$. Poor temporal sampling places few constraints on the timescales of the variability, but in the case of W61a significant changes occur over a few tens of days (Fig. 3). Morel et al. (2004) monitored 12 
J. S. Clark et al.: Stellar variability in Wd1

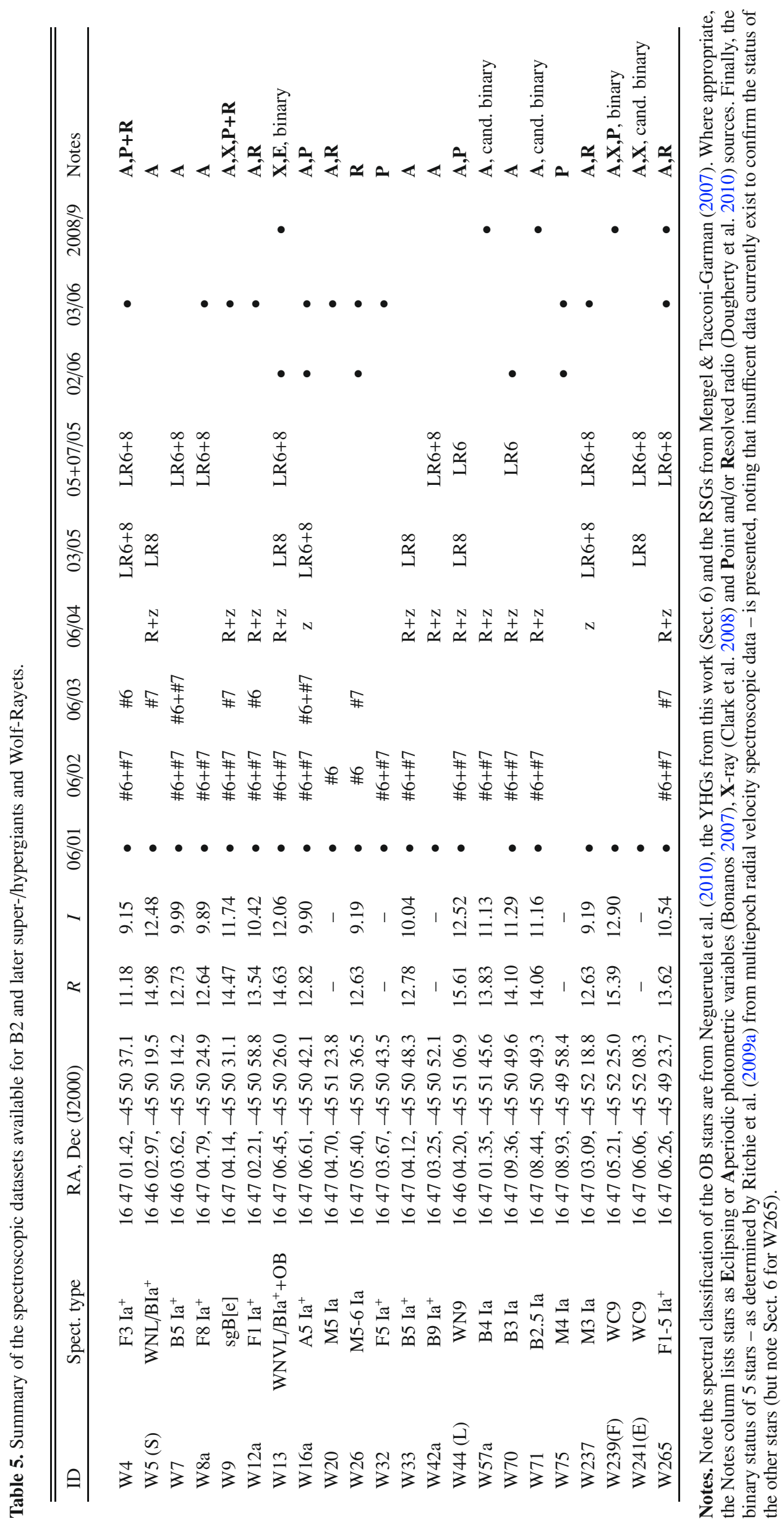



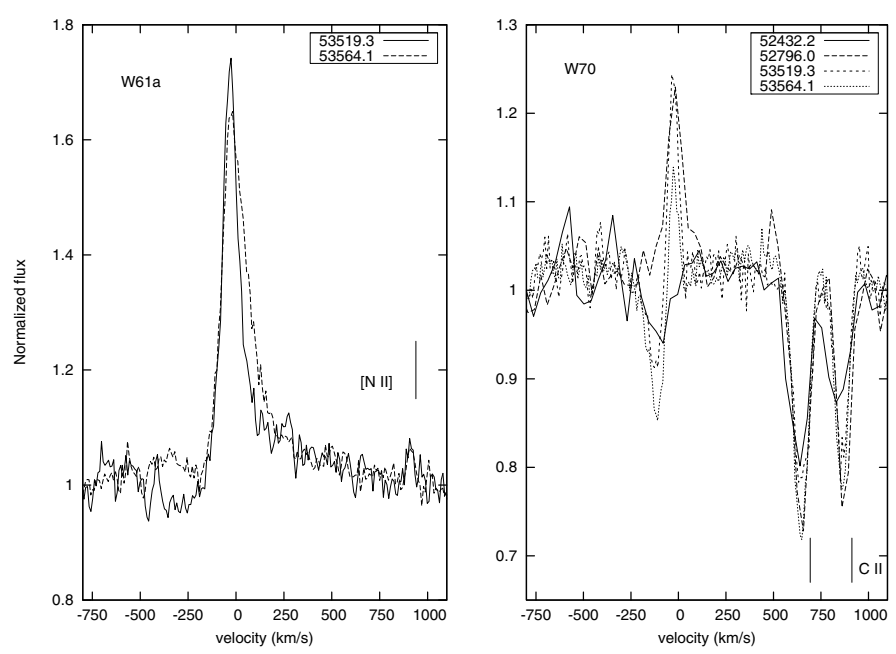

Fig. 3. LPV in $\mathrm{H} \alpha$ for W61a (B0.5 Ia) and W70 (B3Ia). Note the variability in the strength of the temperature dependent $\mathrm{C}$ II photospheric lines in W70.

O9-B2 Ia-Ib field supergiants, finding conspicuous variability on comparably rapid timescale in many of the stars with $\mathrm{H} \alpha$ profiles dominated by wind emission, including - as found for W19 - a transition from absorption to full emission in HD 37128 (B0 Ia). Moreover all of the $\mathrm{H} \alpha$ line profile morphologies demonstrated by the OB supergiant population within Wd1 were found to have identical counterparts in the samples presented in Kaufer et al. (1996) and Morel et al. (2004).

As such the variability of the Wd1 members does not appear atypical for late O/early B supergiants, while the poor sampling frequency of the current dataset suggests that many more stars are likely to be intrinsically variable. Such a supposition is supported by all 9 stars displaying $\mathrm{H} \alpha$ LPV being drawn from the subset of stars with 2 or more epochs of high resolution data. Although is not expected that such stars will have evolved far enough to encounter the LBV phase, the physical origin of such variability is currently uncertain, with pulsational, magnetic and/or binary modulation of the wind all possible. In this respect it is of interest that W2a, 6a, 23a, 30a and 43a have all been identified as binaries (Clark et al. 2008, Negueruela et al. 2010; Ritchie et al. 2009a).

Of the 9 stars with both NTT (2002) and VLT (2004) $I$ band spectra, only one star - W24 - showed evidence for variability; intriguingly this was most pronounced in the C III+Pa16 $8500 \AA$ blend rather than in other stronger, unblended Paschen lines. Motivated by this we examined the full VLT/FLAMES 2008-9 spectroscopic dataset (with analysis of the comparable datasets for the full sample presented in a future dedicated paper). These data confirmed that the blend varied in both strength and radial velocity (RV) of the profile centroid over short timescales $(\sim 24 \mathrm{~d}$; Fig. 4). Given the weakness of the adjacent $\mathrm{Pa} 16$ transition and Pa15+Ca II \& Pa13+Ca II blends as well as the lack of comparable LPV in the Pa11 line we associate this behaviour with the C III $8500 \AA$ A line rather than Pa16 or Ca II $\lambda$ 8498. The physical origin of this behaviour remains uncertain, with no periodicity or systematic secular evolution present in the current data. Ritchie et al. (2009a) report similar LPV for the Paschen series in the OB supergiants W8b, 21, 23a and 78, which they attribute to photospheric pulsations; it is tempting to include W24 in this category, although why pulsations should solely be visible in C III $8500 \AA$ and not in the Paschen series is unclear.
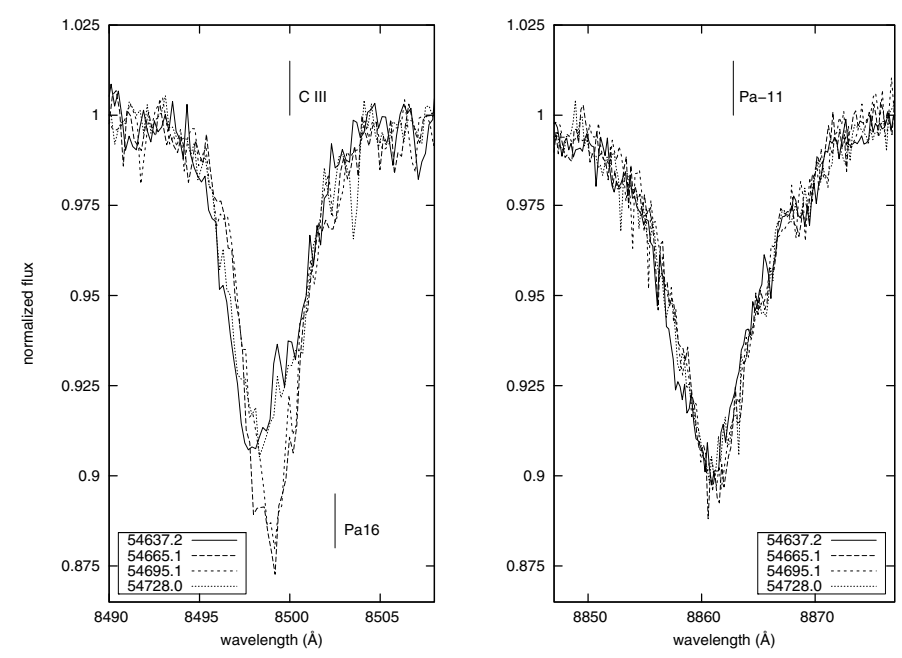

Fig. 4. LPV in the 2008-9 VLT/FLAMES spectra C III+Pa16 blend in W24; in comparison little variability is observed in the unblended Pa11 profile. Note that the strength of C III line in the 2002 NTT and 2004 VLT spectrum, which are not shown for clarity, are comparable to the strong (e.g. MJD 54665.1) and weak (e.g. MJD 54637.2) "states" respectively.

Another group of spectroscopically variable stars are the rare Of?p objects, in which the phenomenon may be linked to the presence of strong magnetic fields (e.g. Nazé et al. 2008). The defining feature of these stars is (variable) emission in the C III $\lambda \lambda$ 4647,50,51 multiplet ${ }^{6}$. Unfortunately this feature is currently inaccessible in W24, and while it is a hard X-ray source it is not over luminous for its bolometric luminosity as might be expected for an Of?p star (Clark et al. 2008). Nevertheless, given the presence of a magnetar within Wd1 (Muno et al. 2006) and the suggestion that these are the descendents of highly magnetised progenitors, the possibility that W24 could be a member of such a population is intriguing and warrants further investigation.

Regarding variability amongst the OB supergiant population within Wd1, we may also utilise the results of Negueruela et al. (2010) to reinterpret the findings of Bonanos (2007). In Fig. 5 we plot a semi-empirical HR diagram for all the OB supergiants within Wd1 for which a spectral classification is available, highlighting the location of the photometric variables identified in the latter work. This emphasises that such stars are predominantly concentrated amongst the more luminous, evolved SGs; a similar result to that found by Fullerton et al. (1996). Given the apparent dependence on evolutionary state we assume that the photometric variability is most likely intrinsic to the star and hence not binary in origin.

It is tempting to associate this behaviour with the $\alpha$ Cygni variables, of which the subset of late O/early B supergiants demonstrate quasi-periodic oscillations with $\leq 0.1 \mathrm{mag}$ amplitude (e.g. van Leeuwen et al. 1998). Unfortunately, the observations of Bonanos (2007) - comprising two 10 day blocks separated by 20 days - are ill-suited to identifying the photometric modulation of $\alpha$ Cygni variables, which characteristically occur over 2-6 week timescales for early OB supergiants, extending to hundreds of days for cooler super-/hypergiants (van Leeuwen et al. 1998).

\footnotetext{
${ }^{6}$ Note that the C III $8500 \AA$ line is the singlet counterpart $\left(3 s^{1} \mathrm{~S}-3 \mathrm{p}^{1} \mathrm{P}^{0}\right)$ to this multiplet.
} 


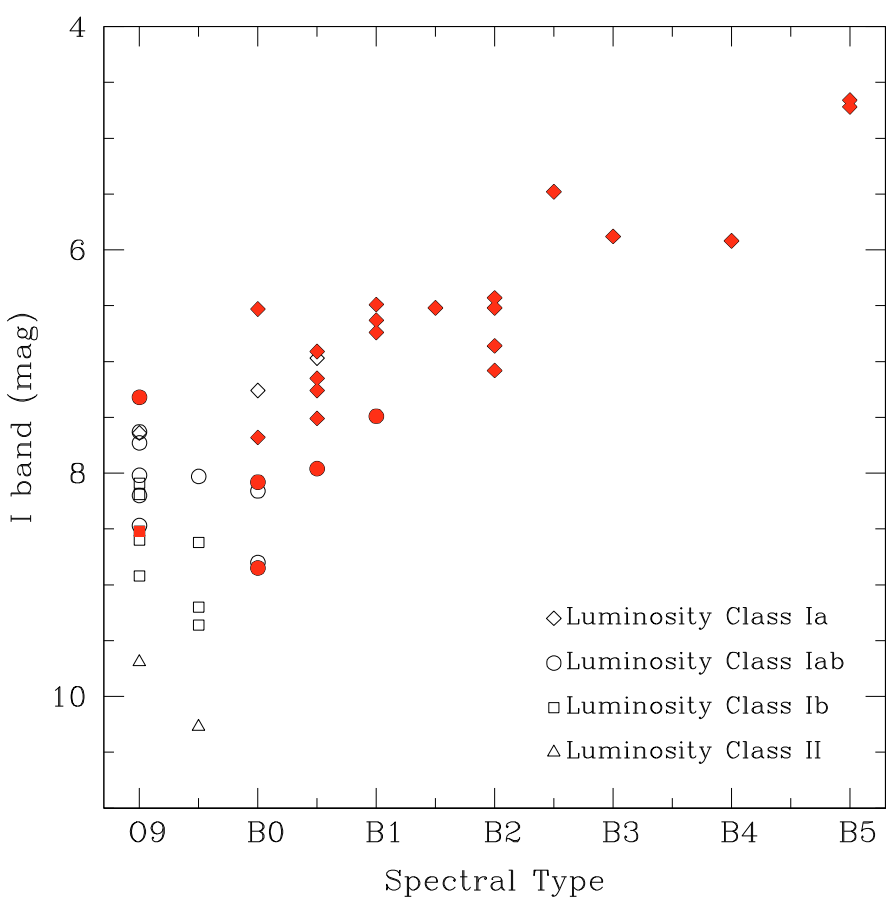

Fig. 5. Plot of $I$ band magnitude versus spectral type for the late $\mathrm{O}-$ mid B stars within Wd1 (stars individually dereddened according to the prescription given in Negueruela et al. 2010). The photometric variables identified by Bonanos (2007) are indicated by the filled symbols.

Finally, four stars - W6a, 10, 11 and 28 - were also observed by West87; given their spectral classifications agree to $\leq 2$ subtypes we find no evidence for their long term secular evolution. Nevertheless, of the 23 stars considered here, we find that all but four are either spectroscopic or photometric variables (Table 4).

\section{The mid-late $B$ super-/hypergiants}

The 6 stars with spectral types B2.5-9 naturally divide into two subgroups. The first, containing W57a, $70 \& 71$, are earlier (B2.5-4 Ia) and have comparatively weak $\mathrm{H} \alpha$ emission, while the latter, comprising $\mathrm{W} 7,33 \& 42 \mathrm{a}$, are later $\left(\mathrm{B} 5-9 \mathrm{Ia}^{+}\right)$, and demonstrate stronger $\mathrm{H} \alpha$ emission with broad wings (likely due to electron scattering). Despite this, all were found to be spectroscopically variable.

Of the B2.5-4 Ia stars, the $\mathrm{H} \alpha$ profile of $\mathrm{W} 70$ was observed in absorption in 2002, full emission in 2003 and P Cygni emission in 2005 (Fig. 3). The C II $\lambda \lambda 6578,6582$ transitions also appeared to vary in both strength and the RV of the line centroid between observations, with changes in the profiles of both species observed over the shortest timescale probed $(45 \mathrm{~d})^{7}$. In comparison, the early B supergiants HD 14134 (B3Ia) and HD 43384 (B3Iab) also showed a rapid ( $\leq 14 \mathrm{~d}$ ) transition from $\mathrm{H} \alpha$ absorption to emission (Morel et al. 2004), although neither they nor the other B2-4 supergiants from that study demonstrated variability in the $\mathrm{C}$ II lines. These transitions are temperature dependent and are absent in supergiants earlier than B0.7 and later than B9 and peak at B3, although their apparent abundance dependance have precluded their use as an accurate diagnostic (Negueruela et al. 2010). Hence we may conclude that both wind and photosphere

7 While we regard the detection of RV changes as provisional, given the relatively low $S / N$ and resolution of the observations, the changes in the line strength are robust, amounting to $\sim 15 \%$ in Equivalent Width between the identical resolution 2005 FLAMES observations.
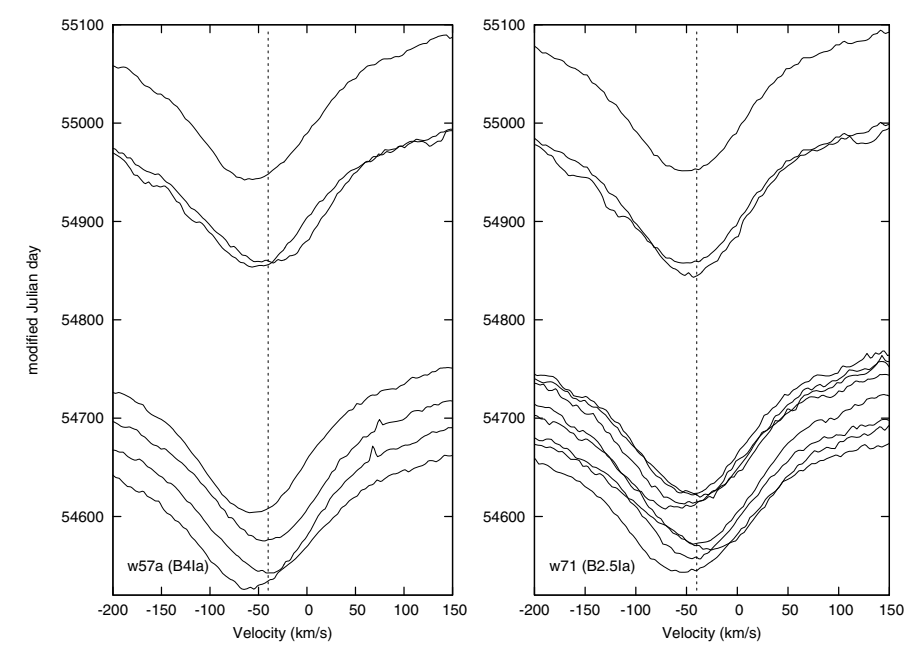

Fig. 6. LPV in Pa11 indicative of photospheric pulsations for the BHGs W57a (B4 Ia) and W71 (B2.5 Ia). Similar variability is also present in the N I lines.

are variable, although we may not constrain the magnitude of the temperature excursions exhibited by the latter.

W71 also demonstrated significant LPV in $\mathrm{H} \alpha$, which was present in emission in 2002 but absent in 2004, while it was absent in both spectra of W57a. For both objects the $S / N$ and resolution of the spectra were too low to comment on the behaviour of the nearby $\mathrm{C}$ II and He I features and for that reason are not shown here. Included in the binary survey (Ritchie et al. 2009a), the multiple $I$ band observations of both stars demonstrated rapid RV variability in the Paschen series and NI lines (Figs. 6-7). Building on these results we found $\Delta \mathrm{RV} \sim 12 \mathrm{kms}^{-1}$ and $25 \mathrm{~km} \mathrm{~s}^{-1}$ for W57a and 71 respectively with no period apparent in either dataset; consistent with random sampling of a short (pulsational) quasi-period, comparable in both respects to the photometric pulsations observed in other early and late B supergiants (Kaufer et al. 1997, 2006). The change in strength of the N I lines in both stars indicates that pulsations were present, being inconsistent with an origin for the RV changes in binarity alone. These imply moderate variations in the spectral type corresponding to $\Delta T \sim 2000 \mathrm{~K}$, noting that modeling of the nonradial pulsations of the B supergiant star HD64760 revealed a comparable difference between minimum and maximum surface temperature (3500 K; Kaufer et al. 2006).

The cool B hypergiants W7, 33 and 42a also demonstrated pronounced $\mathrm{H} \alpha \mathrm{LPV}$, although an emission component was always observed (Figs. 8-9). The C II doublet was also found to be variable in W7; this behaviour was mirrored in the Paschen series and low excitation metallic lines present in the $I$ band spectra (Fig. 10). Variations in both the strength and RV of the C II doublet have previously been observed for the B8 Iae star HD 199478 (Markova et al. 2008); the similarity of the resultant spectroscopic period to the photometric period providing strong evidence for photospheric pulsations in this star. Identical LPV is also present in the $I$ band spectra of W42a (not shown for brevity); in conjunction with comparable behaviour in both hotter and cooler supergiants within Wd1 (W70 and 265 respectively) we conclude that both W7 and W42a are also pulsating. As with W57a and 71, the range of wavelengths sampled by these spectra do not include particularly sensitive temperature diagnostics, although changes in spectral type by \pm 1 subtype may be inferred for both W7 and W42 over timescales of $~ 35$ days (Fig. 10). 

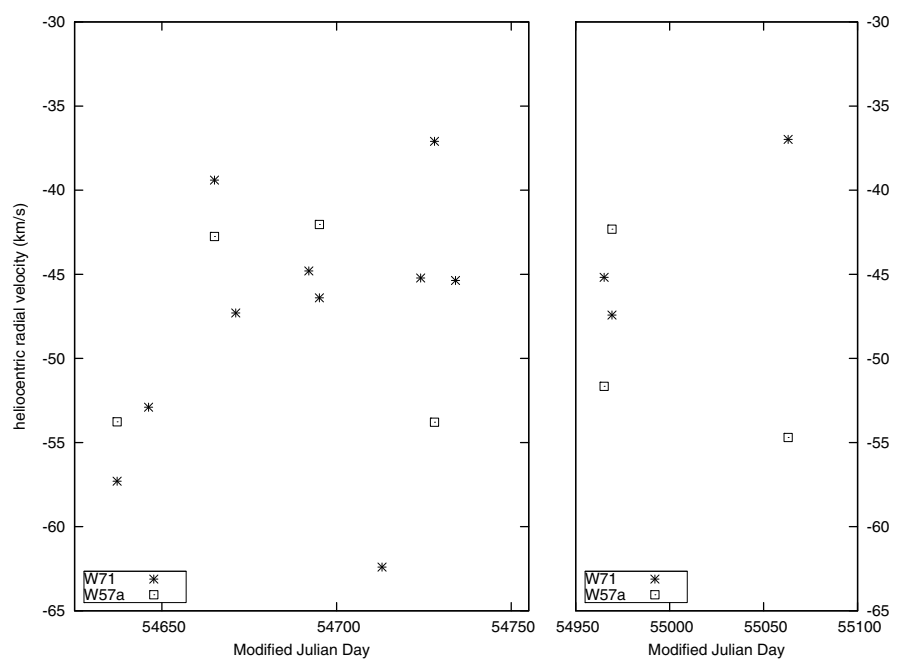

Fig. 7. Plot of $R V$ variations versus time for the Pa11 line in the BHGs W57a (B4 Ia) and W71 (B2.5 Ia). The RV is the error-weighted average of the Pa-11..14 lines, with an uncertainty of $\pm 3 \mathrm{~km} \mathrm{~s}^{-1}$.
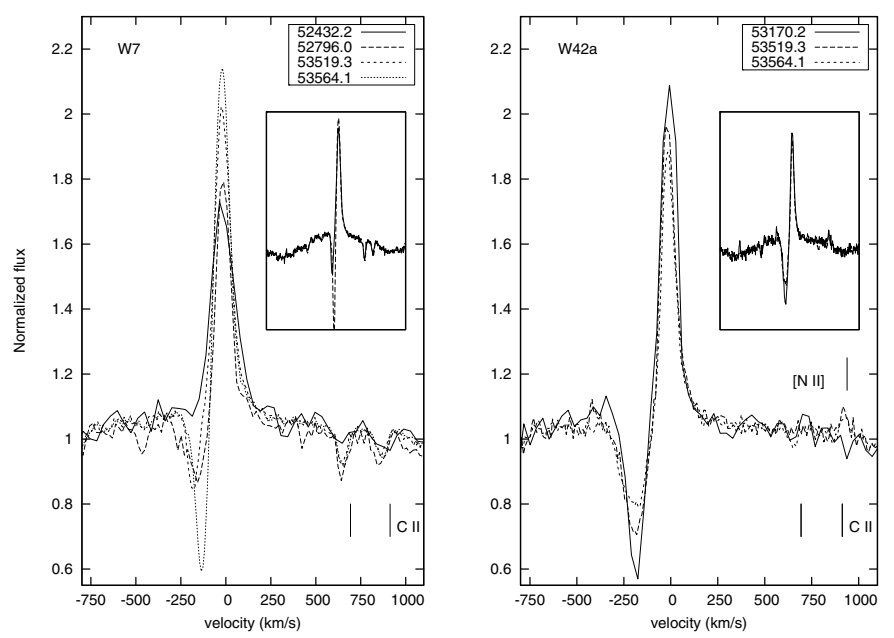

Fig. 8. $\mathrm{LPV}$ in the $\mathrm{H} \alpha$ line of $\mathrm{W} 7\left(\mathrm{~B} 5 \mathrm{Ia}^{+}\right)$and $\mathrm{W} 42 \mathrm{a}\left(\mathrm{B} 9 \mathrm{Ia}^{+}\right)$. The insets show the Halpha profile with a log flux scale to highlight the broad emission wings.

However, as with the B2.5-4 Ia supergiants, the physical origin of the $\mathrm{H} \alpha$ variability is unclear. Comparable P Cygni profiles and LPV in $\mathrm{H} \alpha$ were found for HD 199478, HD 92207 (A0 Ia; Kaufer et al. 1996), and the LBV HD 160529 (Stahl et al. 2003). Both HD 199478 and HD 92207 undergo short lived (20-60 d) episodes of enhanced blueshifted absorption leading to the appearance of a P Cygni profile (Kaufer et al. 1996; Markova et al. 2008); a timescale comparable to that inferred for the LPV in our stars ( $\leq 45$ days). Such "high velocity absorption" (HVA) events are assumed to be due to a transient increase in the optical depth along the line of sight in an aspherical wind, although the origin of the asphericity is unspecified. However, in W7, 33 and 42a the increase in strength of the P Cygni absorption trough was accompanied by an increase in the strength of the emission component of the line. Such correlated behaviour is not observed in HD 199478 and HD 92207, but is seen in the LBV HD 160529 (Stahl et al. 2003), where it is instead attributed to a long term secular change in the global properties of the stellar wind such as mass loss rate and terminal velocity. Further observations are
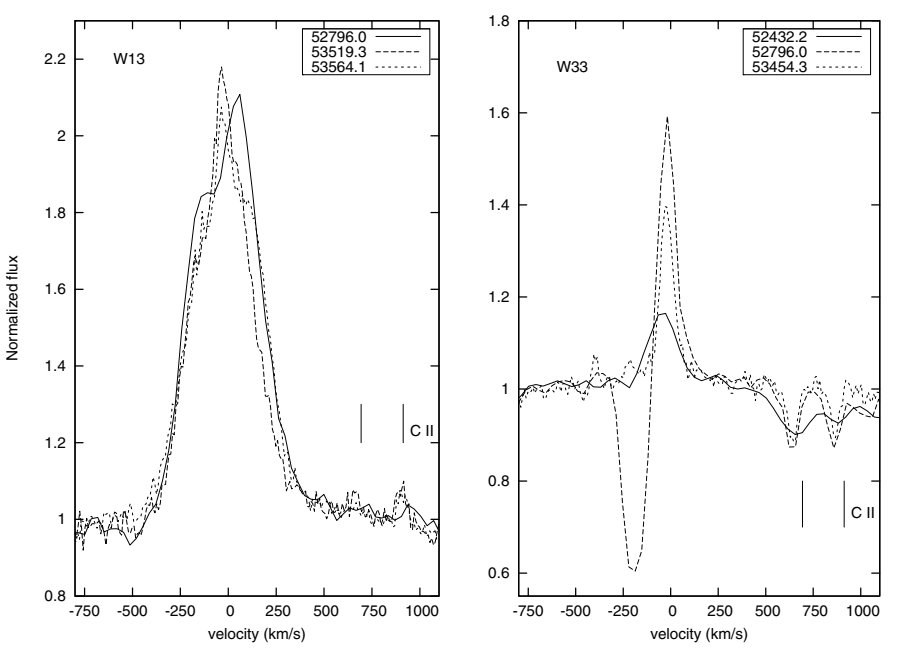

Fig. 9. LPV in the $\mathrm{H} \alpha$ for $\mathrm{W} 13\left(\mathrm{WNVL} / \mathrm{B} \mathrm{Ia}^{+}+\mathrm{OB}\right)$ and $\mathrm{W} 33\left(\mathrm{~B}^{2} \mathrm{Ia}^{+}\right)$.

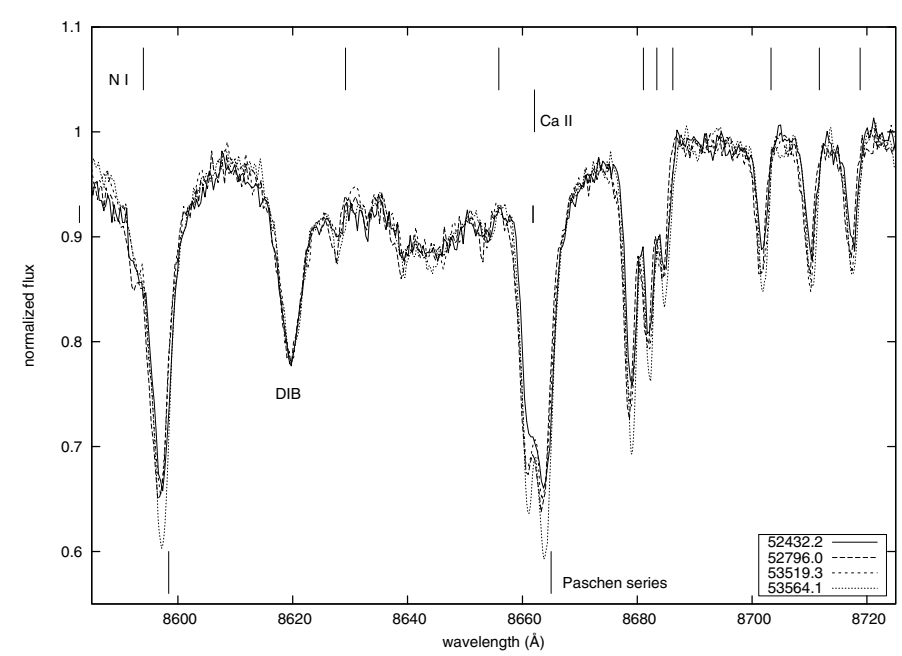

Fig. 10. Pulsational variability in the Paschen series and low excitation metallic lines of $\mathrm{W} 7$ over timescales as short as 35 days.

clearly required to distinguish between both possibilities for the B hypergiants within Wd1.

In summary, evidence for both photospheric (pulsational) and wind variability are found for both sub-groups of B super/hypergiants, although no causal link between both phenomena may be drawn from the current data nor physical cause for wind variability inferred. However, with regard to this we note that no binary markers are found for any of these stars; likewise there is no unambiguous evidence for either long term secular evolution or canonical LBV excursions over the 5-9 yr observational baseline for individual stars (Table 5$)^{8}$. Such a result is not unprecedented; P Cygni has demonstrated only weak secular evolution since its outburst in 17th Century - which would have been undetectable in our data - with strong (>1 mag.) LBV excursions also absent (Lamers \& de Groot 1992; de Groot et al. 2001).

8 I band spectra for LBVs in both high and low temperature phases are provided by Ritchie et al. (2009b) and Munari et al. (2009), while observations of both early and late B/early A supergiants - the range over which a putative LBV within Wd1 might be expected to evolve - are presented in C05 and Munari \& Tomasella (1999). These data clearly demonstrate that changes in the spectral morphology resulting from a canonical LBV excursion would be identifiable in our data. 
Likewise the B hypergiants VI Cyg 12 and $\zeta^{1}$ Sco - which show a remarkable spectral similarity to the BHGs within $\mathrm{Wd} 1$ - have also been photometrically stable for a century (Clark et al. in prep.; Sterken et al. 1997).

In relation to this, the spectral types determined by West 87 and $\mathrm{C} 05$ for $\mathrm{W} 7,42 \mathrm{a}, 70$ \& 71 are discrepant (Table 2). However given the difficulties of $I$ band classification (Negueruela et al. 2010) we strongly suspect these arise from the low $S / N$ and resolution of the spectra presented by West 87 rather than from any intrinsic long term variability. In conjunction with photometric classifications (Table 2) we may therefore also exclude any long term transition to a significantly higher or lower temperature for these stars, such as the decades long excursions seen for R127 and M33 Var A (Walborn et al. 2008, Humphreys et al. 2006; Sect. 6).

The final OB supergiant star we turn to is the $\operatorname{sgB}[\mathrm{e}] \mathrm{star}$ W9. Given their co-location in the HR diagram, there has been much speculation on the evolutionary links between $\mathrm{sgB}[\mathrm{e}]$ stars and LBVs. Both photometric (e.g. Bergner et al. 1995; Zickgraf et al. 1996) and spectroscopic (e.g. Zickgraf 2003) variability have been observed in these stars but to date these have not been unambiguously associated with characteristic LBV excursions or eruptions. In this respect W9 is of interest, given the evidence for changes in its unexpectedly high mass loss rate (Dougherty et al. 2010) and the detections of short term aperiodic photometric fluctuations (Bonanos 2007). A full description of the optical and IR spectroscopic dataset is presented in Ritchie et al. (submitted) but, perhaps unexpectedly, we found no evidence for pronounced spectroscopic variability over the course of the observations (2001-4; noting the comparatively low resolution data would not resolve LPV similar to that observed in other hot supergiants).

\section{The Wolf Rayets}

WRs have long been implicated in the LBV phenomenon; in their hot, quiescent state LBVs such as AG Carinae display a WN9-11 spectral morphology, while the WN3 star HD 5980 underwent a major eruption resulting in a transition to significantly cooler spectral types in the 1980s (WN6 and

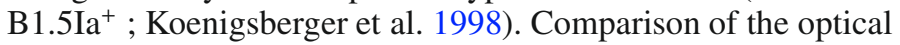
and near-IR spectroscopic datasets (Clark \& Negueruela 2002; Negueruela \& Clark 2005; Crowther et al. 2006) indicate that no such extreme behaviour is evident for any of the WRs within Wd1 between 2001-5, with the discrepancies between the subtypes of individual objects being attributable to a combination of low $S / N$ and resolution spectra and a poorly callibrated $I$ band classification scheme. Unfortunately, the faintness of the WR population limits the utility of the long term photometry; of the 8 stars observed on two or more epochs ${ }^{9}$, only W44 (=WR L) is potentially variable.

As with $\mathrm{OB}$ supergiants, line profile variability has been observed in a large number of both WN and WC WRs (e.g. Robert 1994; Lepine \& Moffat 1999), typically taking the form of a superposition of variable subpeaks on an underlying broad emission line and attributed to both small (stochastic clumping) and large scale (co-rotating) wind structure. The origin of the latter is uncertain, with both photospheric and binary origins advanced for the quasi-periodic line profile variability observed in e.g. EZ CMa (=WR6; Morel et al. 2004; Georgiev et al. 1999), although apparently unambiguous binary modulation has been

9 WRs A, B, E, F, G, L, M \& S

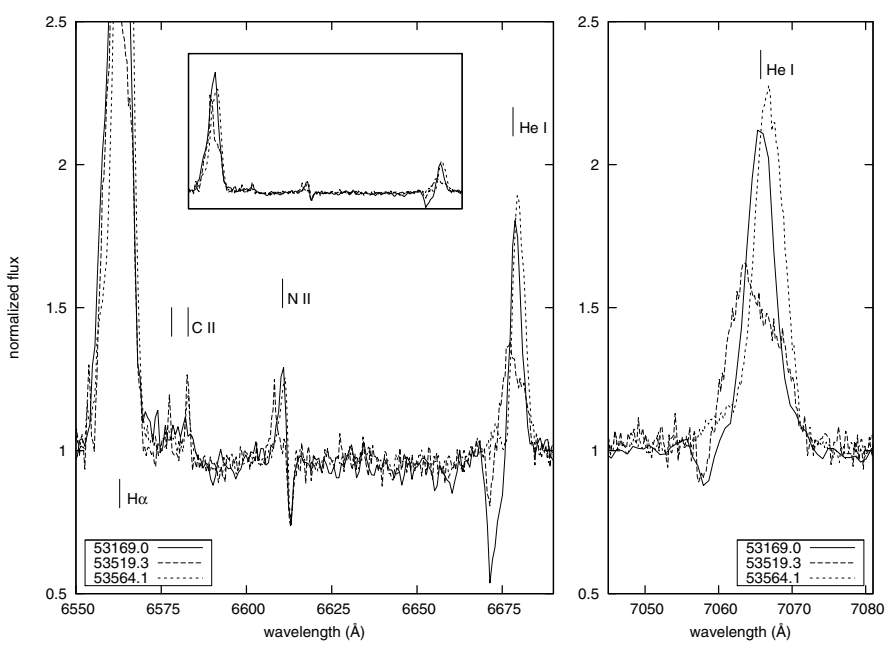

Fig. 11. LPV in $\mathrm{H} \alpha$ and He I transitions for the candidate WN9 binary W44. The inset in the left panel shows the same wavelength range but rescaled in flux to show the whole $\mathrm{H} \alpha$ profile.

reported in a number of WR colliding wind systems by Stevens \& Howarth (1999).

Including the extreme B hypergiants W5 (=WR S) and W13, which show a close continuity in physical properties to the WN9 star W44, a total of five WRs have multiepoch optical spectroscopic data, the others are the WC9 stars W239 (=WR F) and W241 (=WR E). Negueruela et al. (2010) present the highest resolution and $S / N I$ band spectrum of W5 available to us; no evidence for long term line profile variability between 2001-4 was visible in these data. W13 is discussed in depth in Ritchie et al. (2009a), who showed it to be a B Ia ${ }^{+} / \mathrm{WNVL}+\mathrm{OB}$ binary. In addition to reflex RV motion in the Paschen series, significant variability was present in the emission components of profiles obtained at comparable orbital phases, behaviour replicated in the $\mathrm{H} \alpha$ profile (Fig. 9). Upon consideration of the full dataset no secular evolution was apparent in any spectral feature.

Likewise, comparison of optical data from 2001-5 showed no long term changes for the WN9 star W44. However high resultion spectra revealed dramatic LPV in $\mathrm{H} \alpha$ and $\mathrm{He}$ I $\lambda \lambda 6678$, 7065 (Fig. 11) which, to the best of our knowledge, are more pronounced than the variations observed for any other WR star outside of an LBV excursion. Given their absence in the spectra of our cool B hypergiants, the continued presence of strong $\mathrm{He} \mathrm{I} \lambda, \lambda$ 6678,7065 emission throughout this period argues against attributing the changes to a transition to a cool LBV phase. On the basis of its X-ray properties W44 is a strong CWB candidate (Clark et al. 2008) and despite the above concerns - and lack of photometric confirmation - as with W13 we consider binary induced wind structure to be the most likely explanantion for the spectral behaviour, but further data are required to confirm this hypothesis.

Finally we turn to the WC stars W239 and W241 (Fig. 12). Ritchie et al. (2009a) showed that W239 demonstrated reflex binary motion between $2008-9$ but, while intrinsically variable, the expanded dataset revealed no evidence for secular evolution between 2001-9. Spectra of the dusty WC9 star W241 in 2005 May and July demonstrated notable changes in the strengths of the He I+II and C II+III lines and following the arguements for W44 we suspect these too are due to binarity. Of interest are a number of sharp emission features superimposed on the broad C II emission line at $\sim 6580 \AA$. We suspect these reflect emission 

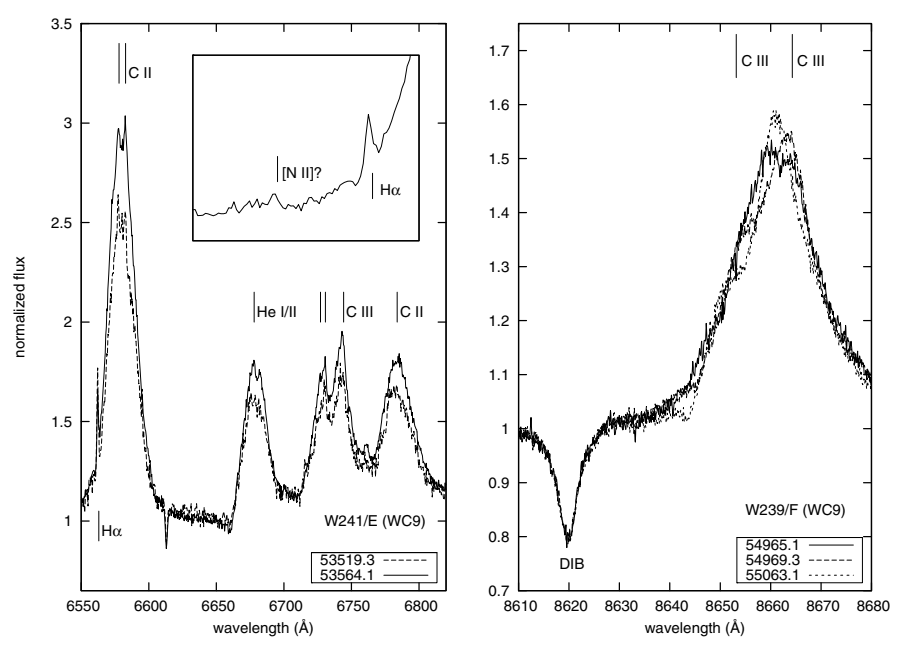

Fig. 12. Variability in the spectra of the WC9d stars W239 and W241. Note the sharp emission features superimposed on the broad C II emission line of W241, with a possible identification of the $[\mathrm{N} \mathrm{II}] \lambda 6548$ line in the blue wing (indicated in the inset).

in $\mathrm{H} \alpha$ and $\mathrm{C} \operatorname{II} \lambda \lambda 6578,6582$, with a further, tentative identification of emission in the $[\mathrm{N}$ II] $] \lambda 548$ line also possible (the stronger $[\mathrm{N} \mathrm{II}] \lambda 6584$ line is potentially blended with $\mathrm{C}$ II $\lambda$ 6582). The origin of such lines is currently unclear. We would not expect narrow $\mathrm{H} \alpha$ or $[\mathrm{NII}]$ emission to be associated with a WC star, suggesting they must originate in the hitherto unseen companion. However, given the current data we are unable to determine whether the emission lines are nebular in origin or instead whether they arise in a stellar wind; if the latter is correct they would imply a rather early spectral type for the secondary.

\section{The yellow hypergiants}

As with the hotter B hypergiants, field YHGs are also highly variable over multiple timescales. Most, if not all, demonstrate short period pulsations with periods of the order of $10^{2}$ days (e.g. de Jager 1998 and refs. therein) visible in both lightcurve and RV variations in photospheric lines; similar rapid variability is also present in wind contaminated emission lines such as $\mathrm{H} \alpha$ (e.g. Lobel et al. 2003). Additionally, long term secular temperature variations have been inferred from both spectroscopic and photometric observations for a number of the most luminous YHGs. Oudmaijer et al. (1996) found that IRC +10 420 increased in temperature by $>1000 \mathrm{~K}$ over the past twenty years, with similar behaviour inferred for HD 179821 (Patel et al. 2008). In contrast HR 8752 has undergone two red loops in the HR diagram over the past thirty years, each of approximately 10 years duration (de Jager \& Nieuwenhuijzen 1997). Similar excursions to cool temperatures have been observed for $\rho$ Cas (Lobel et al. 2003) although the duration of these events is significantly shorter ( $\leq 2$ yrs).

Two or more observations of the $\mathrm{H} \alpha$ profile are available for 5 of the 6 YHGs within Wd1. Of these we infer $\mathrm{H} \alpha$ to be variable in W265 (reported to be present by West87 but absent in our data), observe significant evolution in the profile of W16a (Fig. 13; apparently absent in West87) and find no variability for $\mathrm{W} 4,8 \mathrm{a}$ and $12 \mathrm{a}^{10}$. While only 3 epochs of spectroscopy

\footnotetext{
10 Only a single epoch of high resolution and $S / N$ data is available for $\mathrm{W} 12 \mathrm{a}$ - as such we would not expect to detect the subtle $\mathrm{H} \alpha \mathrm{LPV}$ observed for e.g. $\rho$ Cas (Lobel et al. 2003).
}

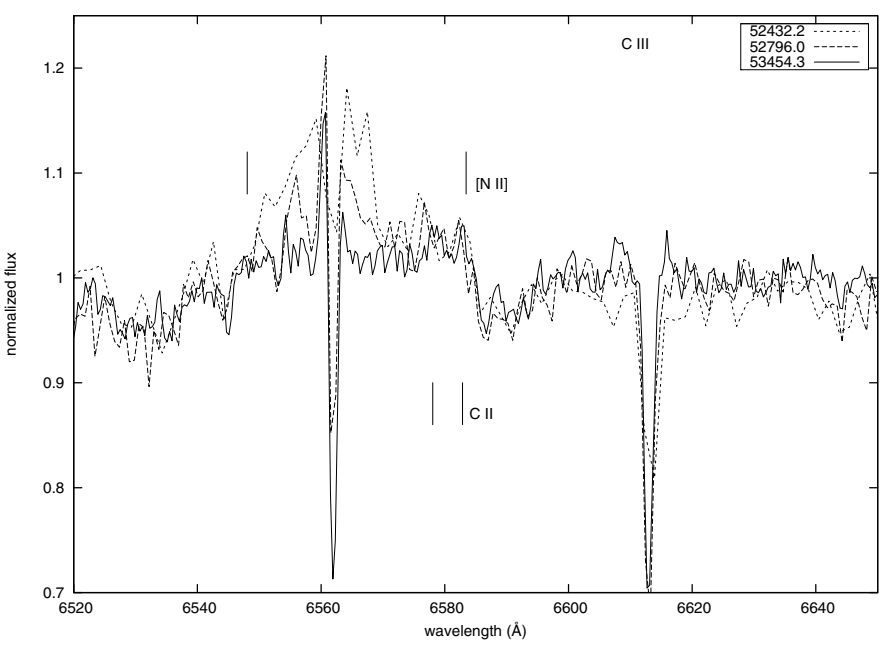

Fig. 13. LPV in $\mathrm{H} \alpha$ in W16a (A5 $\mathrm{Ia}^{+}$) between 2002-5. Note the match between the strengths of the DIB in the 2003 \& 2005 spectra implies that the change in strength of the narrow emission and absorption components is real. The location of C II $\lambda \lambda 6578,82$ and [N II] $\lambda 6583$ are also indicated.

are available for W16a, the profile appears to show a number of unusual features. The first, a narrow blueshifted emission component superimposed on a presumably photospheric absorption profile is also present in the YHGs HD 179821 (Patel et al. 2008) and $\rho$ Cas (Lobel et al. 2003) where, like here, it is variable. A broad plinth of emission extends to some $\pm 1000 \mathrm{kms}^{-1}$ and appears constant in all three spectra, while a third emission component extending from $\sim-700 \mathrm{~km} \mathrm{~s}^{-1}-+300 \mathrm{~km} \mathrm{~s}^{-1}$ seems to be present in the 2002 spectrum but absent in 2005 ; to the best of our knowledge this combination of features is not present in any other YHG.

Pronounced emission wings are present in the mid-late B hypergiants W7 and 42a, as well as lower luminosity late B/early A field supergiants (Kaufer et al. 1996). Typically, such features are thought to arise from electron scattering of line photons but no strong emission is present in W16a, nor in several of the stars studied by Kaufer et al. (1996). Moreover the high velocity emission plinth is asymmetric in W16a and is more pronounced in the red, although this might be due to blended emission in the coincident [N II] $\lambda 6583$ and/or $\mathrm{C}_{\text {II }} \lambda \lambda$ 6578, 6582 lines (noting that the later appear unlikely given they are only seen in the extreme early B supergiants). This feature is also reminiscent of the He I $\lambda \lambda$ 5876, 6678 lines in W9 (Clark et al. 2008), which Ritchie et al. (2010) tentatively attribute to the presence of a wind collision zone. However, there is no evidence that W16a is a CWB; therefore we are currently unable to offer a convincing physical explanation for either the line morphology or variability of $\mathrm{H} \alpha$ in W16a.

Preliminary observations of the neutral metallic lines of W265 by Ritchie et al. (2009a) suggested that it was pulsating; the inclusion of a second season of observations allows us to observe the turnaround in the pulsational cycle, implying a quasi-period of order $\sim 10^{2}$ days (Figs. 14-15). Unlike the late B/early A supergiants studied by Kaufer et al. (1997), we find a depth dependence for the velocity fields of the photospheric lines, with the higher excitation lines - which form deeper in the atmosphere - showing less variability than the lower excitation transitions, although all lines vary in phase. Comparison of the spectra taken at the extremes of this cycle to the classification standards of Munari \& Tomasella (1999 Fig. 16) show it to vary 

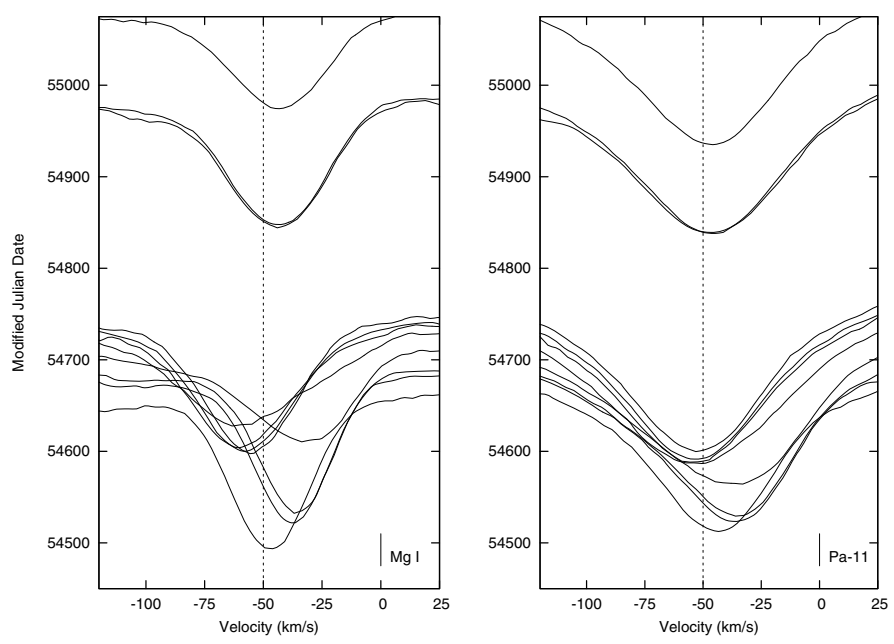

Fig. 14. Pulsations in the photospheric Mg I and Pa11 lines in W265.

between spectral types F0 (7200K) and F5 Ia ${ }^{+}(6570 \mathrm{~K})$, spending most time in the cool phase.

The magnitude of this change is directly comparable to that observed for $\rho$ Cas in observations between 1993-5 ( $\Delta \mathrm{T} \sim 750 \mathrm{~K}$; Lobel et al. 1998), although the 2000-1 outburst of $\rho$ Cas, which resulted in a greatly enhanced mass loss rate, was accompanied by a more extreme evolution of spectral type than observed here (early M supergiant; Lobel et al. 2003). Moreover, (i) the timescale of quasi-pulsations (mean value 300 days; de Jager et al. 1998 and refs. therein); (ii) the average $\Delta \mathrm{RV} \sim 18 \mathrm{~km} \mathrm{~s}^{-1}$ for low excitation (1-5 eV) photospheric lines (Lobel et al. 1994); (iii) the development of excess absorption in the blueshifted wings of both the $\mathrm{Ca}$ II and Fe I lines during the hot phase (due to enhanced mass loss leading to an increased optical depth in an expanding atmosphere; Lobel et al. 1998, Ritchie et al. 2009a) and (iv) the significant velocity stratification in photospheric lines originating at different atmospheric depths (Lobel et al. 2003) in $\rho$ Cas are all directly comparable to the same phenomena in W265. Thus both stars appear to demonstrate similar pulsationally driven mass loss, presumably via the same physical mechanism.

Unfortunately, the spectral datasets available for $\mathrm{W} 4,8 \mathrm{a}, 12 \mathrm{a}$ and $16 \mathrm{a}$ are of insufficient resolution and $S / N$ to readily identify futher pulsators via RV shifts in the photospheric lines, although variations in the strength of neutral metallic lines are present in $\mathrm{W} 4$, and hence by analogy to W243 and 265, provide evidence for pulsations. Given the relatively poor temporal sampling of these observations and the finding that W265 appears to spend most of its time in the cool phase, we suspect that the remaining stars may also turn out to be variable. Nevertheless these data do permit accurate spectral classification ${ }^{11}$; we found W4 $(=\mathrm{F} 3$ $\left.\mathrm{Ia}^{+}\right)$, W8a $\left(=\mathrm{F} 8 \mathrm{Ia}^{+}\right), \mathrm{W} 12 \mathrm{a}\left(=\mathrm{F} 1 \mathrm{Ia}^{+}\right)$and W16a $\left(=\mathrm{A} 5 \mathrm{Ia}^{+}\right)$to be systematically later by $2-3$ subtypes than found by $\mathrm{C} 05$ utilising lower resolution data. Given classification of A-G supergiants is primarily accomplished via the relative strengths of weak

\footnotetext{
11 Interestingly, some evidence exists for the presence of abundance anomalies in these data. NI absorption features are significantly enhanced in W12a with respect to W265; given little temperature dependence is expected for these lines between $\mathrm{F} 1-5 \mathrm{Ia}^{+}$we suspect this is the result of chemical processing. Likewise photospheric $\mathrm{C}$ I absorption lines are present in all these stars but are absent in the cool-phase LBV W243 (Ritchie et al. 2009b), suggesting C depletion in the latter object. A full analysis of these data will be presented in a future paper.
}
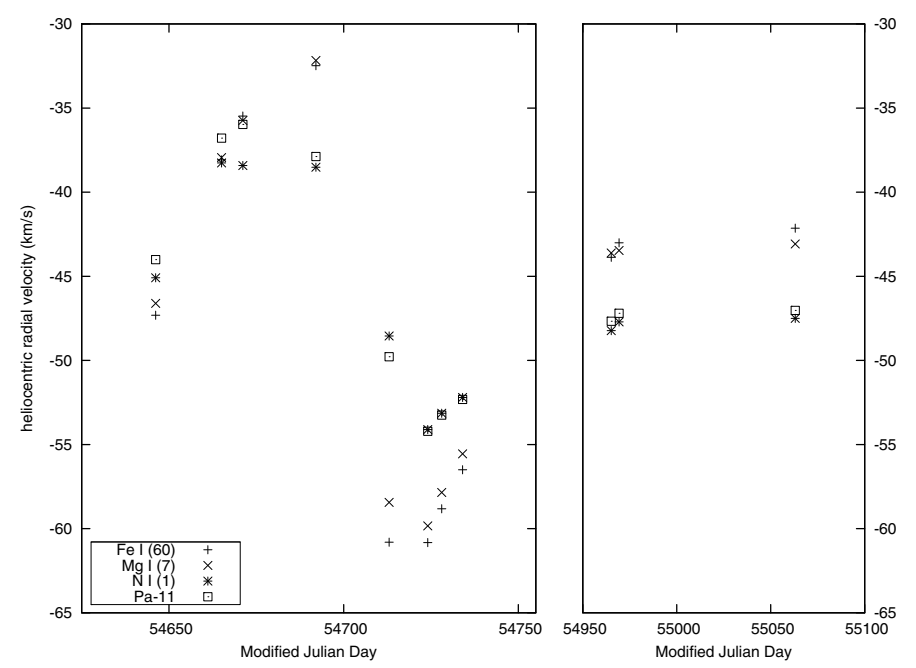

Fig. 15. Plot of the evolution of the RV of selected photospheric line in W265; included are the upper photospheric lines of Fe I (multiplet 60, $\chi_{\text {low }} \sim 2.2 \mathrm{eV}$ ), $\mathrm{Mg}$ I (multiplet $7, \chi_{\text {low }} \sim 4.3 \mathrm{eV}$ ), N I (multiplet 1 , $\left.\chi_{\text {low }} \sim 10.2 \mathrm{eV}\right)$ and the Pa11 line $\left(\chi_{\text {low }} \sim 12.04 \mathrm{eV}\right)$.

metallic lines we conclude that this discrepancy results from the different quality spectra available rather than being of astrophysical origin. A similar conclusion may also be drawn for the systematically later classifications of W4, $8 \mathrm{a}, 32$ and 265 by West 87 (Table 2). The classifications for W12a appear noticeably discrepant; we suspect this is due to an erroneously early spectral type in West87; the strength of the Pa18+O I, Pa16+Ca II and Pa15+Ca II blends are all directly comparable to W265 in his data, as we find in ours.

Consequently, we find no spectroscopic evidence for long term secular evolution of the temperature of any of the YHGs over the quarter of a century between 1981-2005. Such a conclusion is bolstered by the photometry available; the $\sim$ magnitude visual brightening found for IRC +10 420 between 1930-70 (Humphreys et al. 1992) would have been detectable if similar had occured for any YHG between 1966-2006 (Sect. 2). However, given the poor temporal sampling we may not exclude shorter events such as the red loops and outbursts which HR 8752 and $\rho$ Cas have been observed to undergo (de Jager 1998, Lobel et al. 2003), although long lived excursions to cool temperatures, such as seen for M33 Var A - for which a transition from an $\mathrm{F}$ to $\mathrm{M}$ supergiant, accompanied by a 3 mag decrease in the $V$ band, persisted for $\sim 45$ years (Humphreys et al. 2006) would have been detected.

\section{The red supergiants}

Finally we turn to the RSGs W20, 26, 75 \& 237, supplementing our sparse optical dataset (Table 5) with the near-IR classifications of Mengel \& Tacconi-Garman (2007). West87 report $\mathrm{H} \alpha$ emission in W20 \& 26 and its absence in W237, with no data for W75. We fail to detect $\mathrm{H} \alpha$ in W20, but weak emission is present in W237, although at a level that could have been missed in West87 (Fig. 17); by analogy with W26, we consider it likely to be a blend of $\mathrm{H} \alpha+[\mathrm{N}$ II] . Variable line emission in the Balmer series (and low excitation species) has previously been observed in the RSG+OB binary VV Cephei stars (Cowley 1969), where it is thought to arise in the region of the RSG wind ionised by the hot companion. 


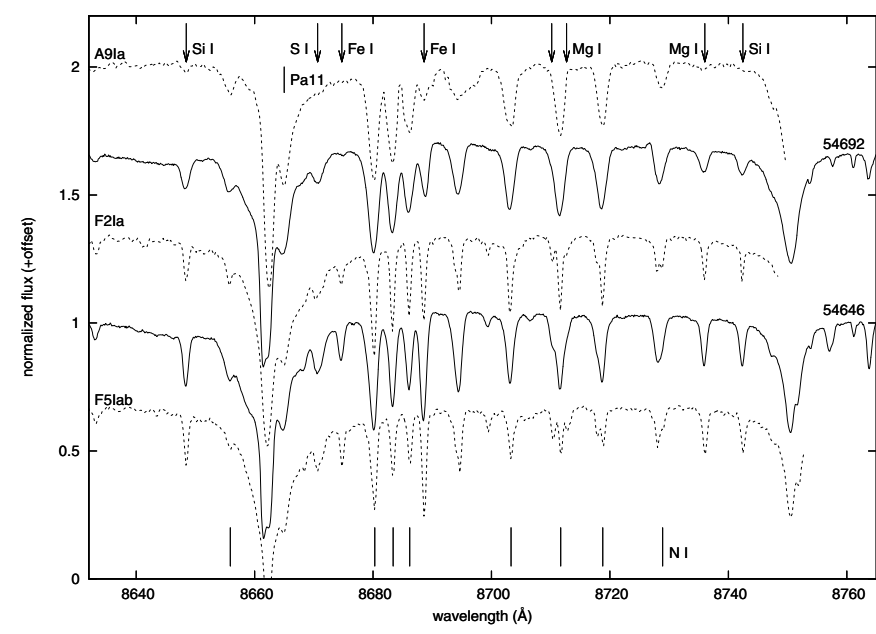

Fig. 16. Comparison of spectra of W265 taken in the hot and cool phase to the classification spectra of Munari \& Tomasella (1999).

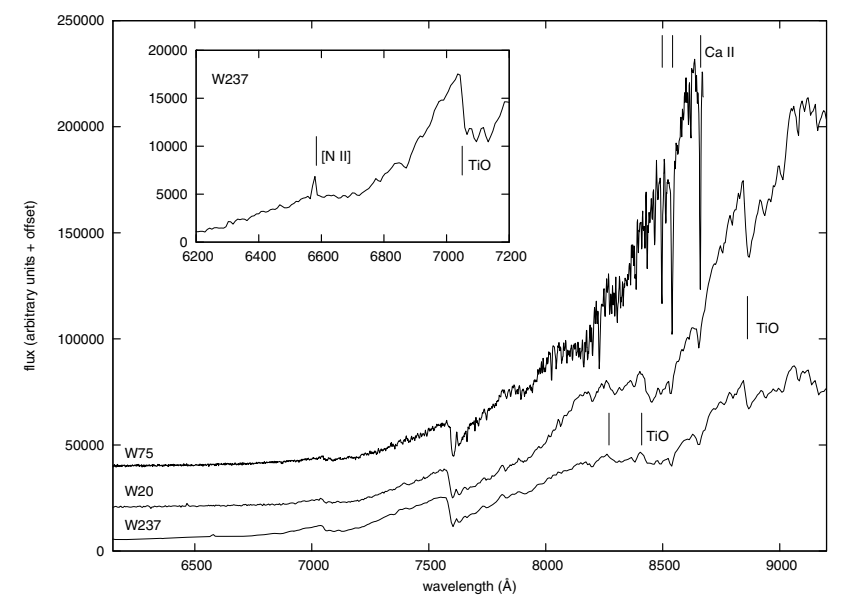

Fig. 17. Low resolution spectra of the RSGs W20 \& 237 from 2001 June and a medium resolution spectrum of W75 from 2006 February, with an inset centred on the $\mathrm{H} \alpha+[\mathrm{N} \mathrm{II}]$ blend in W237. Note that no emission is present in this feature in $\mathrm{W} 20$, while the $S / N$ is insufficient to comment for W75.

In contrast W26 continues to demonstrate strong $\mathrm{H} \alpha$ emission in our data (Fig. 18), as well as forbidden line emission from [N II] and [S III]; given the strength of the former with respect to $\mathrm{H} \alpha$ we suspect this emission arises in nitrogen enriched ejecta rather than a stellar wind. No changes are apparent in the strength of the $\mathrm{H} \alpha+[\mathrm{N} \mathrm{II}]$ emission in W26 between 2001-6, although the missmatched resolutions mean that we are insensitive to subtle LPV. To the best of our knowledge only one other RSG - WOH G64 - is known to show such a pronounced nebular emission line spectrum (Levesque et al. 2009; see Appendix A for more details).

No line diagnostics for spectral type are present within the wavelength regions sampled by our spectra and so we are forced to rely on the strength of the TiO bandheads at $\sim 7050, \sim 8250$, $\sim 8440$ \& $\sim 8860 \AA$, which increase in strength with decreasing temperature (e.g. Levesque et al. 2005; Zhu et al. 1999; Ramsey et al. 1981). Unfortunately, the low resolution and $S / N$ complicate an absolute classification for the spectra from 2001, but we note that the bandhead appears significantly weaker in W26 than W20 and W237, suggesting an earlier spectral type for the

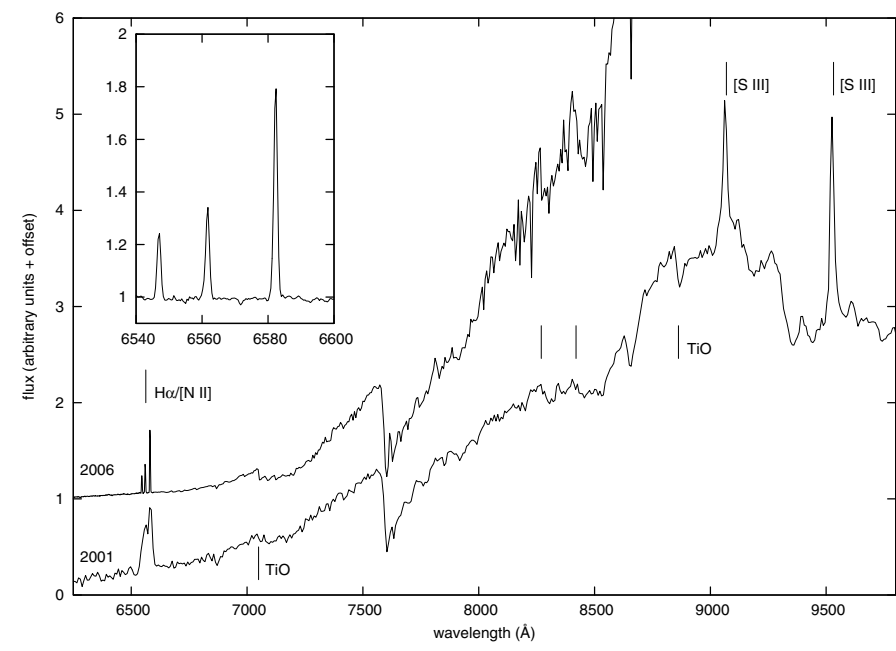

Fig. 18. Spectra of W26 from 2001 June and 2006 February respectively; note that the resolution of the 2006 spectrum has been artificially degraded to better match that of the 2001 data. The TiO bandheads are significantly stronger in the latter spectrum, indicative of a later spectral type. The inset shows the region of the spectrum from 2006 centred on $\mathrm{H} \alpha$ at the original resolution.

former, while the strengths of these features in W20 \& 237 clearly differ (Figs. 17 and 18).

Higher resolution spectra of the $\sim 8860 \AA$ bandhead for W20, W26 (both 2002 June) and W237 (2004 June; identical to those from 2005 March-July) are presented in Fig. 19. These indicate that W26 is of unambiguously earlier spectral type than W20 and W237, which in these data - unlike in the 2001 spectra are indistinguishable from one another. We therefore may conclude that the temperature of one or both of these stars has varied between the two observations. Based on the callibration of the $\sim 8860 \AA$ bandhead by Ramsey et al. (1981) we may assign spectral types of M2 Ia for W26 and M5 Ia for W20 and 237, with an uncertainty of \pm 1 subtype in absolute rather than relative classification. An additional spectrum of W26 from 2006 February (Fig. 18) suggests a significantly later spectral type at this time in comparison to previous spectra (no earlier than M4 Ia from the strength of the $\sim 8440 \AA$ bandhead; Zhu et al. 1999), while our sole spectrum of W75 from the same run appears to be much earlier than any other RSG (no later than M0 Ia; Fig. 17).

Given the different near-IR diagnostics employed by Mengel \& Tacconi-Garman (2007) a straightforward comparison of their classifications to those derived from these data is difficult. However, they find W20 and W26 to be of comparable spectral type (M5 Ia) and later than W237 (M3 Ia), with their classification of W26 being compatible with ours from the previous month.

We therefore conclude that W26 appears to unambiguously demonstrate changes in spectral type (M2-5 Ia), with pronounced variations in the $\mathrm{TiO}$ bandhead diagnostics between 2001-6. W237 also appears to be spectroscopically variable based its classification relative to W20 and W26, with a comparison of the photometry presented by C05 and Bonanos (2007) supporting this assertion (Sect. 2; we cannot exclude the possibility that W20 is also variable). Finally, we simply note the discrepancy between optical ( $<$ M0 Ia) and near-IR (M4 Ia) classifications of W75 between February-March 2007.

Both photometric and spectroscopic variability may be present in high luminosity RSGs, although the latter is thought to be particularly rare (e.g. Levesque 2009). The extreme RSG 


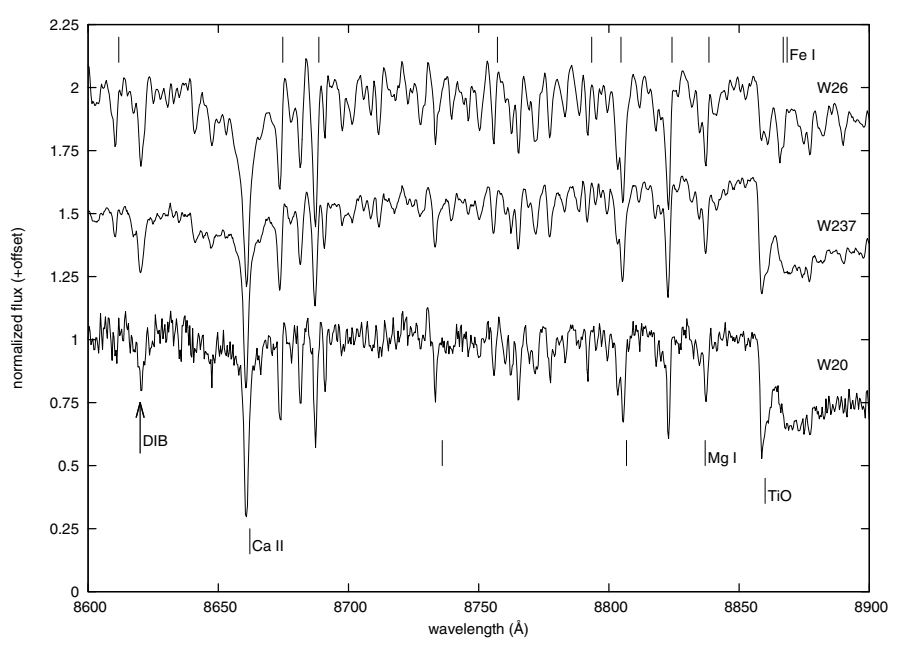

Fig. 19. High resolution $I$ band spectra of the RSGs W20, 26 (2002 June) and 237 (2004 June) covering the diagnostic TiO bandhead at $8860 \AA$ A.

VX Sgr demonstrates "semi-regular variations" between M5.5$9.8 \mathrm{Ia}$, accompanied by $\Delta \mathrm{V}>6 \mathrm{mag}(\Delta \mathrm{I} \sim 1.2 \mathrm{mag})$ over a $\sim 732$ day quasi-period (Lockwood \& Wing 1982), while S Per transited from M4-7 Ia between 1971-4 (Fawley et al. 1977) and has also undergone pronounced photometric variability ( $\sim 6 \mathrm{mag})$ over the past century (Chipps et al. 2004). Significant evolution in this behaviour is also seen; between 1889-1919 VY CMa underwent irregular variations with $\Delta V<1 \mathrm{mag}$. and a mean quasi-period of $600 \mathrm{~d}$, after which the excursions increased in both magnitude ( $\Delta V \sim 3 \mathrm{mag}$ ) and duration ( 1100 d; Robinson 1970).

Given the inevitable constrains imposed by our limited datasets, we conclude that the variability of W26 and W237 is entirely consistent with the behaviour of these extreme RSGs, implying that they too are unstable. Given the quasi-periodic nature of the lightcurves of VX Sgr, SPer and VY CMa, Lockwood $\&$ Wing (1982) liken this behaviour to a high luminosity analogue of the pulsations that are present in Mira variables. The presence of substantial circumstellar ejecta around three of the four RSGs within Wd1 (Dougherty et al. 2010) which, in the case of W26, appears to be nitrogen enriched, supports the assertion of Massey et al. (2007) that this instability may be associated with significant mass loss. Finally, as with both the BHGs and YHGs, the long term photometry (Table 2) precludes any long term excursions analagous to that undergone by M33 Var A.

\section{Discussion and concluding remarks}

Despite the disparate nature of the photospheric and spectroscopic dataset accumulated for $\mathrm{Wd} 1$ over the past 50 years, it presents a valuable resource to investigate the properties of the massive stellar population and in particular to characterise the prevalence and nature of stellar variability. This is of particular relevance given the unique census of evolved stars within Wd1, comprising examples of all post-MS stellar types thought to originate from stars with $M_{\text {initial }} \sim 30-40 M_{\odot}$. Given the limited sampling of the combined dataset it was somewhat surprising to find that stars in all evolutionary states were variable, with this behaviour being attributable to both evolving wind structure(s) and stellar pulsations. Indeed these phenomena appear to be commonplace, with 26 of the OB super-/hypergiants and all but one of both the cool hypergiants and the Wolf Rayets included in this study being either photometrically and/or spectroscopically variable.

Wind variability was most evident in the $\mathrm{H} \alpha$ line of the $\mathrm{OB}$ super-/hypergiants, with the line transiting from absorption to emission in W70, and pronounced P Cygni profiles developing in several other stars. Similar behaviour has been observed in field stars although, as with this population, the physical mechanism(s) driving the wind inhomogineities are poorly understood. In this respect the detection of both photospheric pulsations and wind variability in the mid B supergiants W70 \& 71 and hypergiants W7 \& 42a is of considerable interest and all four stars would benefit from further observations with a higher cadence to investigate potential causal links between the two phenomena (cf. Markova et al. 2008). Moreover, these results again emphasise the uncertainty inherent in determining mass loss rates for $\mathrm{OB}$ supergiants from a single epoch of $\mathrm{H} \alpha$ spectroscopic data.

Significant wind structure is also inferred for the WRs investigated. Given the identification of binary markers such as the presence of hot dust, hard X-ray emission and, in the case of W13 and 239, reflex RV variations, we attribute this to the effect of the companion via wind/wind interaction, noting that no secular evolution is present in any of the stars. This is encouraging since it suggests that detailed modeling of these variation may yield the underlying binary properties of the systems (e.g. Stevens \& Howarth 1999) enabling a comparison to the less evolved stellar populations within $\mathrm{Wd} 1$, hence illuminating the effects of binary interaction driven by stellar evolution.

Wind diagnostics are more difficult to identify and study in the cool hypergiants. Nevertheless variable high velocity absorption in the blue wing of the metallic photospheric lines is present in W265, indicative of changes in the optical depth and hence physical properties of the wind. By analogy to $\rho$ Cas, we attribute this to pulsationally driven mass loss. Variability in the $\mathrm{H} \alpha$ emission line profile is inferred for W12a and directly observed in W16a, although the morphology of this line is complex and hence the physical origin of this behaviour is consequently uncertain.

Variations in both profile strength and the RV of the line centroid were found for the photospheric lines of both the hot and cool evolved stellar populations, the combination indicative of pulsations rather than binary motion (e.g. Ritchie et al. 2009a). Amongst the OB supergiants within Wd1, this behavour had previously been reported for B0.5-2 Ia stars; our results extend this to encompass the full spectral range present (O9-B4 Ia). Utilising the new spectroscopic callibrations of Negueruela et al. (2010) we find that the presence of photometric variability (Bonanos 2007) appears to be a strong function of evolutionary phase, being more prevalent amongst the early-mid B Ia stars in comparison to the late O Iab-II stars. This is qualitatively similar to the conclusions drawn by Fullerton et al. (1996) regarding the occurence and magnitude of photospheric pulsations in OB stars and hence it is tempting to attribute the photometric variability to photospheric pulsations, such as those inferred for the $\alpha$ Cygni variables. Unfortunately, we currently lack the high $S / N$ and cadence observations that would enable pulsational mode identification.

Amongst the cooler super-/hypergiants, pulsations were inferred for stars with spectral types ranging from mid B through to mid F. The best studied example - W265 - was observed to transit between $\mathrm{F} 0-5 \mathrm{Ia}^{+}$over the course of a single cycle ( $\sim 100$ days); the combination of both timescale, magnitude and atmospheric depth velocity stratification of the pulsations being strongly reminiscent of those observed in $\rho$ Cas, which have been attributed to non-radial modes (Lobel et al. 1994, 2003). 
Although the data are sparse, both W26 and 237 display spectroscopic variability indicative of changes in spectral type, with the former being observed to evolve from M2-5 Ia. The finding that at least two of the four RSGs within $\mathrm{Wd} 1$ are unstable and by analogy with other extreme RSGs, possibly pulsating is of considerable interest given (i) their extreme luminosity and hence high progenitor mass ${ }^{12}$; (ii) their rich circumstellar envelopes, which provide evidence for significant recent mass loss, possibly driven by these instabilities; and (iii) the rarity of this phenomenon (with only six other examples known in the Galaxy and Magellanic Clouds; Levesque 2009).

Indeed, these preliminary findings emphasise the important role studies of young massive clusters with multiplexing spectrographs will play in understanding the physics of high mass stars. In particular the detection of photospheric pulsations extending from spectral types as early as O9 I, through F5 $\mathrm{Ia}^{+}$hypergiants and potentially as late as the M5 Ia supergiants confirms the finding of e.g. Burki (1978) that stars in this region of the HR diagram are unstable, but critically for a rich co-eval population of stars at a single metallicity rather than a heterogeneous field population. Given the sample size $-\sim 80 \sim$ O8III-B2 Ia stars - future analysis of the full 2008-9 VLT/FLAMES dataset will provide valuable observational constraints on the location of the high temperature boundary of this region of instability in a statistically robust manner.

Furthermore, observational determination of the amplitude and period of the pulsations as a function of temperature, luminosity and evolutionary phase will provide direct contraints on the underlying physical mechanism(s) driving this instability - for example strange - and/or g-modes (Kiriakidis et al. 1993; Saio et al. 2006) - and, in conjunction with chemical abundance analysis, a detailed picture of the evolution of the internal structure of massive stars as they execute a red loop on their way to becoming Wolf Rayets. Similarly, high candence observations will enable an investigation into the role pulsations play in both the origin of wind structure in hot supergiants and in driving mass loss in the cool hypergiants. Indeed, the uniquely rich population of both hot and cool super-/hypergiants within Wd1 holds open the possibility of investigating the varying contribution of both line and pulsational driven mass loss as a function of stellar temperature and luminosity as stars evolve across the HR diagram.

Relating to this, with the exception of the known LBV W243 (Ritchie et al. 2009b), we find no compelling evidence for other LBV like excursions or eruptions within Wd1, despite both the large number of transitional stars present and the evidence for past episodes of enhanced mass loss amongst a subset of both hot and cool stars (Dougherty et al. 2010). H $\alpha$ LPV similar to that observed in the LBV HD160529 was found for several of the B super-/hypergiants, but the poor temporal coverage meant that we could not determine whether these corresponded to the long term secular changes that characterise LBV excursions, or the short lived variations indicative of an asymmetric wind.

However, while similar limitations also apply to the (non-) detection of events comparable to the outbursts of $\rho$ Cas and the red loops of HR8752 in the YHG population, we would have expected to detect events comparable to the multi-decade excursions of e.g. M33 Var A, or the long term secular evolution of

\footnotetext{
12 A lower limit to their progenitor masses may be inferred from the cluster Main Sequence turnoff. This is an important consideration given that the mass/luminosity degeneracy for RSGs as they execute a red loop prevents such a determination for field stars (Meynet \& Maeder 2003).
}

IRC +10 420. Given these results, it is of interest that a comparable analysis for the WNLh stars - which have been proposed to be quiescent LBVs - within the Galactic Centre and Quintuplet cluster similarly reveals little evidence for significant long term variability (Appendix B). Apparently transitional stars co-located with both "bona fide" LBVs and rapidly evolving cool hypergiants in the HR diagram (e.g. Clark et al. 2005b) can enjoy significant periods of quiescence.

These results are surprising given that the majority of the stars observed within Wd1, from the extreme-B hypergiants through to the RSGs, appear to be low-level variables and only borderline-stable. As such one might naively have expected them to be prey to such large scale instabilities, which presumably contribute to the lack of stars above the HD limit. In this respect the YHGs and RSGs are of particular interest since they are located at the low temperature boundary of the HD limit and hence appear to represent the most massive stars that may exist in this region of the HR diagram at solar metallicities.

Drout et al. (2009) present detailed evolutionary predictions for the physical properties of such stars, which we may test against the population within Wd1. Our new spectral classifications confirm the expectation that YHGs evolving from $\sim 40 M_{\odot}$ stars should have relatively high temperatures $(>6000 \mathrm{~K})$, while the lack of secular variability for any of these stars over a period of $\sim 50 \mathrm{yrs}$ is consistent with the expectation of a relatively long lifetime for this phase $\left(\sim 10^{4}-10^{5} \mathrm{yr}\right)$. However at these luminosities YHGs are not expected to evolve to cooler temperatures, but exactly this evolution is indicated by the presence of 4 RSGs within Wd1. An extention of the redwards loop to encompass a RSG phase would result in a corresponding reduction in the time spent as a YHG. While rapid evolution through the YHG phase might be consistent with the real time increase in stellar temperature observed for IRC +10 420 and HD 179821, the continuing absence of this behaviour from the cool hypergiant population of Wd1 will place increasingly stringent constraints on such a scenario.

Therefore a synthesis of (i) the current physical properties of the evolved stars within Wd1 - such as chemical composition, luminosity, temperature and, where observable, surface gravity; (ii) the relative populations of the different evolutionary classes; and (iii) the characterisation of their (pulsational) instabilities and secular variability has great potential for constraining the physics of massive post-MS evolution.

Finally, regarding the ensemble properties of $\mathrm{Wd} 1$, constraining the occurence and amplitude of the pulsationally (and binary) driven RV changes will be crucial in allowing the accurate determination of dynamical masses for unresolved extragalactic clusters (e.g. Gieles et al. 2009), where the velocity dispersion attributable to such processes may match or even dominate virial motions. Likewise determining the properties of photometric outbursts driven by pulsational instabilities in both hot and cool hypergiants will be important in determining the validity of photometric cluster mass estimates, particularly for the lower range of masses where the presence of such an event may dominate the integrated light.

Acknowledgements. J.S.C. acknowledges the support of an RCUK fellowship. This research is partially supported by the Spanish Ministerio de Ciencia e Innovación under grants AYA2008-06166-C03-03 and ConsoliderGTC CSD2006-70. We thank Otmar Stahl for kindly making his spectra of HD160529 available to us, and Ornette Coleman for inspiration during the writing of this paper. 


\section{Appendix A: The emission line spectrum of the RSG W26}

Of the four RSGs within Wd1, only W26 demonstrates a pronounced nebular emission line spectrum, with emission in $\mathrm{H} \alpha$, [N II] $\lambda \lambda 6548,6582$ and [S III] $\lambda \lambda 9069,9532$. The [S III] lines are common in high temperature $\mathrm{HII}$ regions as well as tracing shocked material. No oxygen emission - such as [O I] $\lambda 6300-$ is seen, while [S III] $\lambda 6312$ is particularly temperature dependent (e.g. Osterbrock \& Ferland 2006) and so its absence while [S III] $\lambda \lambda 9069,9532$ emission is present is explicable if the region is not highly excited. The only other RSG to demonstrate a nebular emission line spectrum is the extreme system WOH G64 which, like W26, is also associated with an extensive dusty circumstellar envelope (Levesque et al. 2009). Unlike W26, WOH G64 lacks [S III] emission although [O I] $\lambda 6300$ and [S II] $\lambda 6713$ are present, as is the high excitation line [O III] $\lambda$ 5007, which our spectra do not reach (Levesque et al. 2009).

Given the lack of many (blue) nebular diagnostic lines we are unable to present quantitative conclusions as to the chemical abundances or excitation mechanism for the emission spectrum. However, by analogy to both WOH G64 and the nebula around the BSG Sher25, we suspect the strength of the [N II] emission implies $N$ enrichment in the circumstellar material, presumably as the result of CNO processing (Levesque et al. 2009). Likewise, while we suspect the [S III] emission is shock excited, we cannot exclude the possibility of excitation by either a hot companion or the diffuse UV radiation field of the cluster; indeed the location of W26 within the "core" of Wd1 suggests that the latter may well play a significant role (cf. Dougherty et al. 2010). Nevertheless, the lack of comparable nebular emission in W20 and 237 is notable, particularly given that these 2 RSGs also possess extended circumstellar envelopes comprising both dust and ionised gas; albeit significant less massive and dense than found for W26 (Dougherty et al. 2010).

\section{Appendix B: Long term stellar variability in the galactic centre and quintuplet clusters}

Comparable variability analyses using published data may also be undertaken for both the Galactic Centre (GC - 6 Myr; Paumard et al. 2006). and Quintuplet clusters ( 4 Myr; Figer et al. 1999). Multiple spectroscopic observations spanning a decade have been made of the emission line star population within the GC cluster (e.g. Najarro et al. 1997; Paumard et al. 2001; Martins et al. 2007) which are complemented by the long term ( decade) photometric datasets of Rafelski et al. (2007) and Trippe et al. (2006). In the absence of cool hypergiants and comparable multiple spectroscopic datasets for the OB supergiant population we restrict the discussion to the narrow line He I stars, which recent analysis by Martins et al. (2007) confirm to be Ofpe/WNL stars. Despite the implication of such stars in the LBV phenomena only one star - IRS34W - was observed to undergo photometic variations of a magnitude comparable to canonical LBV excursions (Trippe et al. 2006), with a second, IRS16NW, being a low level variable ( $\Delta K \sim 0.2 \mathrm{mag})$. However, with the exception of reflex radial velocity motion in the eclipsing binary IRS16SW, no spectroscopic variability was found for any of these stars, including IRS34W (Trippe et al. 2006; Martins, priv. comm. 2009), noting that the $S / N$ and resolution of the spectra would clearly have identified any significant changes in morphology characteristic of the LBV phenomenon (e.g. Clark et al. 2009), as well as the LPV exhibited by e.g. W7 and W33.
Regarding the Quintuplet, both the cool B hypergiants FMM362 and the Pistol star are spectroscopically (Figer et al. 1999; Figer, priv. comm. 2009) and photometrically variable (Glass et al. 1999), suggesting that they are undergoing LBV excursions. Unfortunately, only two epochs of spectroscopy are available for the remaining cluster population, separated by of order a decade (Figer et al. 1999; Liermann et al. 2009). Of these, the former are of significantly lower resolution and $S / N$, although are still sufficient to distinguish between e.g. cool hypergiants such as the Pistol star, hotter Ofpe/WNL and $\sim$ featureless OB supergiant spectra. However, with the exception of FMM362 and the Pistol star, none of the other stars in common between the studies appear to demonstrate unambiguous spectral variability, despite the presence of a number of luminous WN9 and late O/early B supergiants within the Quintuplet. These results are bolstered by Glass et al. (1999) who only found two other confirmed cluster members to be photometrically variable between 1994-7; the dusty WCL stars Q2 and Q3, for which this behaviour may be attributed to time variable dust production.

Thus, somewhat suprisingly, of the 10 WN9 and WN9/Ofpe stars for which multiepoch data exist, none have demonstrated characteristic LBV spectral variability, while only a single object would have been photometrically classified as such. Mindful of the sparse nature of the Quintuplet datasets, these findings are suggestive of a rather long duty cycle for such excursions if such stars are quiescent hot phase LBVs.

\section{References}

Bergner, Y. K., Miroshnichenko, A. S., Yudin, R. V., et al. 1995, A\&AS, 112, 221

Bessell, M. 1986, PASP, 98, 1303

Bonanos, A. Z. 2007, AJ, 133, 2696

Borgman, J., Koornneef, J., \& Slingerland, J. 1970, A\&A, 4, 248

Brandner, W., Clark, J. S., Stolte, A., et al. 2008, A\&A, 478, 137

Burki, G. 1978, A\&A, 65, 357

Castro-Carrizo, A., Quintana-Lacaci, G., Bujarrabal, V., Neri, R., \& Alcolea, J. 2007, A\&A, 465, 457

Chipps, K. A., Stencel, R. E., \& Mattei, J. A., 2004, JAVSO, 32, 1

Clark, J. S., \& Negueruela, I. 2002, A\&A, 396, L25

Clark, J. S., Egan, M. P., Crowther, P. A., et al. 2003, A\&A, 412, 185

Clark, J. S., Negueruela, I., Crowther, P. A. et al. 2005a, A\&A, 434, 949 (C05)

Clark, J. S., Larionov, V. M., \& Arkharov, A. 2005b, A\&A, 435, 239

Clark, J. S., Muno, M. P., Negueruela, I., et al. 2008, A\&A, 477, 147

Clark, J. S., Crowther, P. A., Larionov, V. M., et al. 2009, A\&A, 507, 1555

Cowley, A. P. 1969, PASP, 81, 481

Cranmer, S. R., \& Owocki, S. P. 1996, ApJ, 462, 469

Crowther, P. A., Hadfield, L. J., Clark, J. S., Negueruela, I., \& Vacca, B. D. 2006, MNRAS, 372, 1407

de Groot, M., Sterken, C., \& van Genderen, A. M. 2001, A\&A, 376, 224

de Jager, C. 1998, A\&ARv, 8, 145

de Jager, C., \& Nieuwenhuijzen, H. 1997, MNRAS, 290, L50

de Jager, C., Lobel A., \& Israelian, G. 1998, A\&A, 325, 714

Dougherty, S. M., Clark, J. S., Johnson, T., \& Chapman, J. M. 2010, A\&A, 511, A58

Drout, M. R., Massey, P., Meynet, G., Tokarz, S., \& Caldwell, N. 2009, ApJ, 703, 441

Fawley, W. M. 1977, ApJ, 218, 181

Figer, D. F., McLean, I. S., \& Morris, M. 1999, ApJ, 514, 202

Figer, D. F., MacKenty, J. W., Robberto, M., et al. 2006, ApJ, 643, 1166

Fullerton, A. W., Gies, D. R., \& Bolton, C. T. 1996, ApJS, 103, 475

Georgiev, L. N., Koenigsberger, G., Ivanov, M. M., St-Louis, N., \& Cardona, O. 1999, A\&A, 347, 583

Gieles, M., Sana, H., \& Portegies Zwart, S. F. 2010, MNRAS, 402, 1750

Glass, I. S., Matsumoto, S., Carter, B. S., et al. 1999, MNRAS, 304, L10

Humphreys, R. M., \& Davidson, K. 1994, PASP, 106, 1025

Humphreys, R. M., Jones, T. J., Venn, K., et al. 1992, in Instabilities in evolved super-and hypergiants, North Holland, Amsterdam, ed. C. de Jager, \& H. Nieuwenhuijzen, 80 
Humphreys, R. M., Jones, T. J., Polomski, E., et al. 2006, AJ, 131, 2105

Kaufer, A., Stahl, O., Wolf, B., et al. 1996, A\&A, 305, 887

Kaufer, A., Stahl, O., Wolf, B., et al. 1997, A\&A, 320, 273

Kaufer, A., Stahl, O., Prinja, R. K., et al. 2006, A\&A, 447, 325

Kiriakidis, M., Fricke, K. J., \& Glatzel, W. 1993, MNRAS, 264, 50

Koenigsberger, G., Pena, M., Schmutz, W., et al. 1998, ApJ, 499, 889

Koornneef, J. 1977, A\&A, 55, 469

Krabbe, A., Genzel, R., Eckart, A., et al. 1995, ApJ, 447, L95

Lamers, H. J. G. L. M., \& de Groot, M. J. H. 1992, A\&A, 257, 153

Lewis, D., Moffat, A. F. J., Matthews, J. M., Robert, C., \& Marchenko, S. V. 1993, ApJ, 405, 312

Lepine, S., \& Moffat, A. F. J. 1999, ApJ, 514, 909

Levesque, E. M. 2009, New Astr. Rev., accepted [arXiv: 0911.4720]

Levesque, E. M., Massey, P., Olsen, K. A. G., et al. 2005, ApJ, 628, 973

Levesque, E. M., Massey, P., Plez, B., et al. 2009, AJ, 137, 4744

Liermann, A., Hamann, W.-R., \& Oskinova, L. M. 2009, A\&A, 494, 1137

Lobel, A., de Jager, C., Nieuwenhuijzen, H., Smolinski, J., \& Gesicki, K. 1994, A\&A, 291, 226

Lobel, A., Israelian, G., de Jager, C., et al. 1998, A\&A, 330, 659

Lobel, A., Dupree, A. K., Stefanik, R. P., et al. 2003, ApJ, 583, 923

Lockwood, G. W. 1974, ApJ, 193, 103

Lockwood, G. W., \& Wing, R. F. 1982, MNRAS, 198, 385

Marco, A., \& Negueruela, I. 2009, A\&A, 493, 79

Markova, N., Prinja, R. K., Markpv, H., et al. 2008, A\&A, 487, 211

Martins, F., Genzel, R., Hillier, D. J., et al. 2007, A\&A, 478, 219

Massey, P., Levesque, E. M., Olsen, K. A. G., Plez, B., \& Skiff, B. A. 2007, ApJ, 660,301

Mengel, S., \& Tacconi-Garman, L. E. 2007, A\&A, 466, 151

Mengel, S., \& Tacconi-Garman, L. E. 2008, Ap\&SS, 324, 321

Meynet, G., \& Maeder, A. 2003, A\&A, 404, 975

Morel, T., Marchenko, S. V., Pati, A. K., et al. 2004, MNRAS, 351, 552

Munari, U., \& Tomasella, L. 1999, A\&AS, 137, 521

Munari, U., Siviero, A., Bienatmé, O., et al. 2009, A\&A, 503, 511

Muno, M. P., Clark, J. S., Crowther, P. A., et al. 2006, ApJ, 636, L41

Najarro, F., Krabbe, A., Genzel, R., et al. 1997, A\&A, 325, 700

Nazé, Y., Walborn, N. R., \& Martins, F. 2008, Rev. Mex. Astron. Astrofis., 44, 331

Negueruela, I., \& Clark, J. S. 2005, A\&A, 436, 541

Negueruela, I., Clark, J. S., \& Ritchie, B. W. 2010, A\&A, accepted
Osterbrock, D. E., \& Ferland, G. J. 2006, in Astrophysics of Gaseous Nebulae and Active Galactic Nuclei, second edition (Sausalito, California: Univ. Science Books)

Oudmaijer, R. D., Groenewegen, M. A. T., Matthews, H. E., Blommaert, J. A. D. L., \& Sahu, K. C. 1996, MNRAS, 280, 1062

Owocki, S. P. 2000, Radiatively Driven Stellar Winds from Hot Stars, in Encyclopedia of Astronomy and Astrophysics (London: Nature Publishing Group, and Bristol: Institute of Physics Publishing)

Patel, M., Oudmaijer, R. D., Vink, J. S., et al. 2008, MNRAS, 385, 967

Paumard, T., Maillard, J. P., Morris, M., et al. 2001, A\&A, 366, 466

Paumard, T., Genzel, R., Martins, F., et al. 2006, ApJ, 643, 1011

Piatti, A. E., Bica, E., \& Claria, J. J. 1998, A\&AS, 127, 423

Rafelski, M., Ghez, A. M., Hornstein, S. D., Lu, J. R., \& Morris, M. 2007, ApJ, 659,1241

Ramsey, L. W. 1981, AJ, 86, 557

Ritchie, B. W., Clark, J. S., Negueruela, I., et al. 2009a, A\&A, 507, 1555

Ritchie, B. W., Clark, J. S., Negueruela, I., et al. 2009b, A\&A, 507, 1567

Ritchie, B. W., Clark, J. S., \& Negueruela, I. 2010, A\&A, submitted

Robert, C. 1994, Ap\&SS, 221, 137

Robinson, L. J. 1970, IBVS, 465, 1

Saio, H., Kuschnig, R., Gautschy, A., et al. 2006, ApJ, 560, 1111

Schuster, M. T., Humphreys, R. M., \& Marengo, M. 2006, AJ, 131, 603

Smith, N., \& Gerhz, R. D. 1998, AJ, 116, 823

Stahl, O., Gang, T., Sterken, C., et al. 2003, A\&A, 400, 279

Stevens, I. R., \& Howarth, I. D. 1999, MNRAS, 302, 549

Sterken, C. 1977, A\&A, 57, 361

Sterken, C., de Groot, M., \& van Genderen, A. M. 1997, A\&A, 326, 640

Trippe, S., Martins, F., Ott, T., et al. 2006, A\&A, 448, 305

van Leeuwen, F., van Genderen, A. M., \& Zegelaar, I. 1998, A\&AS, 128, 117

Walborn, N. R., Stahl, O., Gamen, R. C., et al. 2008, ApJ, 683, L33

Westerlund, B. E. 1961, PASP, 73, 51

Westerlund, B. E. 1987, A\&AS, 70, 311

Wood, P. R., Bessell, M. S., \& Fox, M. W. 1983, ApJ, 272, 99

Yamamuro, T., Nishimaki, Y., Motohara, K., Miyata, T., \& Tanaka, M. 2007, PASJ, 59, 973

Zhu, Z. X., Friedjung, M., Zhao, G., Hang, H. R., \& Huang, C. C. 1999, A\&AS, 140,69

Zickgraf, F.-J. 2003, A\&A, 408, 257

Zickgraf, F.-J., Kovacs, J., Wolf, B., et al. 1996, A\&A, 309, 505 


\section{J. S. Clark et al.: Stellar variability in Wd1}

Table 2. Comparison of previously published spectral classifications.

\begin{tabular}{lccccccc}
\hline \hline W\# & \multicolumn{7}{c}{ Spectral classification } \\
& 1966 & 1973 & $1973-4 ?$ & 1981 & 1995 & 2002 & 2006 \\
\hline 4 & $O B$ & $O B$ & - & G0 Ia & $O B$ & F2 Ia & $<$ G5 Ia \\
6 & $O B$ & - & - & B1 V & - & B0.5 Iab & - \\
7 & $O B$ & $O B$ & - & A0 Ia & $O B$ & B5 Ia & - \\
$8 \mathrm{a}$ & $O B$ & - & - & G0 Ia & $O B$ & F5 Ia & $<$ G5 Ia \\
9 & $O B$ & $?$ & $?$ & Be & $O B$ & sgB[e] & $<$ G5 Ia \\
10 & $O B$ & - & - & B0 III & - & B0.5I+OB & - \\
11 & $O B$ & - & - & B1 II & - & B2 Ia & - \\
$12 \mathrm{a}$ & $O B$ & $O B$ & - & A2 Ia & $O B$ & A5 Ia & $<$ G5 Ia \\
$16 \mathrm{a}$ & $O B$ & $O B$ & - & A2 Ia & $O B$ & A2 Ia & - \\
20 & $M$ & $M$ & $M$ & M6 Ia & - & $<$ M6 I & M5 Ia \\
26 & $M$ & $M$ & $M$ & M2 Ia & $M$ & $<$ M6 I & M5-6Ia \\
28 & $O B$ & - & - & B0 II & - & B2 Ia & - \\
31 & $O B$ & - & - & Be & - & B0I+OB & - \\
32 & $O B$ & $O B$ & - & G5 Ia & $O B$ & F5 Ia & $<$ G5 Ia \\
33 & $O B$ & - & - & B8 Iab & $O B$ & B5 Ia & - \\
70 & $O B$ & - & - & B8 Iab & - & B3 Ia & - \\
71 & $O B$ & - & - & B8 Iab & - & B2.5 Ia & - \\
75 & $M$ & $M$ & $M$ & - & - & - & M4 Ia \\
78 & $O B$ & - & - & B1 Ia & - & B1 Ia & - \\
237 & $M$ & $M$ & $M$ & M6 & - & $<$ M6 I & M3 Ia \\
243 & $O B$ & $O B$ & - & B2Ia & - & A2 Ia & - \\
265 & $O B$ & $O B$ & - & G0 Ia & - & F5 Ia & $<$ G5 Ia \\
\hline
\end{tabular}

Notes. Note that classifications given in italics have been derived from photometric data alone, while the 2006 classifications are derived from $2.3 \mu \mathrm{m}$ spectroscopy (Mengel \& Tacconi-Garman 2007, 2008). Crowther et al. (2006) provide a comparable table for the WR population.

Table 4. Summary of spectroscopic data for supergiants of spectral type B2 and earlier.

\begin{tabular}{|c|c|c|c|c|c|c|c|c|c|c|}
\hline ID & $\begin{array}{l}\text { Spectral } \\
\text { type }\end{array}$ & RA, Dec (J2000) & $R$ & $I$ & $\begin{array}{l}1.5 \mathrm{~m} \\
(2001)\end{array}$ & $\begin{array}{l}\text { NTT } \\
(2002)\end{array}$ & $\begin{array}{l}\text { NTT } \\
(2003)\end{array}$ & $\begin{array}{c}\text { VLT } \\
(2004)\end{array}$ & $\begin{array}{c}\text { VLT } \\
(2008 / 9)\end{array}$ & Notes \\
\hline W2a & B2Ia & $164659.7-455051.1$ & 14.23 & 11.73 & $\bullet$ & \#6 & & $\mathrm{R}+\mathrm{z}$ & & $\mathbf{A}$, cand. binary, $\mathrm{H} \alpha$ var. \\
\hline W6a & B0.5Iab & $164704.0-455021.0$ & 15.80 & 13.16 & • & & \#7 & $\mathrm{R}+\mathrm{z}$ & - & $\mathbf{P}$, cand. binary, $\mathrm{H} \alpha$ var. \\
\hline W8b & B1.5Ia & $164706.0-455025.0$ & - & - & & & & $\mathrm{R}+\mathrm{z}$ & - & pulsator \\
\hline W10 & $\mathrm{B} 0.5+\mathrm{OB}$ & $164703.3-455034.7$ & - & - & - & & & $\mathrm{R}+\mathrm{z}$ & & A, binary \\
\hline W11 & B2 Ia & $164702.2-455047.0$ & 14.52 & 11.91 & & $\# 6+\# 7$ & & $\mathrm{R}+\mathrm{z}$ & & $\mathbf{A}$ \\
\hline W15 & O9Ib & $164707.0-455028.0$ & 16.38 & 13.75 & - & & & $\mathrm{R}$ & - & $\mathbf{A}$ \\
\hline W19 & B1Ia & $164704.9-455059.1$ & 15.21 & 12.37 & & $\# 6+\# 7$ & & $R+z$ & & A, $\mathrm{H} \alpha$ var. \\
\hline W21 & B0.5Ia & $164702.0-4551 \quad 11.0$ & 15.56 & 12.74 & & & & $\mathrm{R}+\mathrm{z}$ & - & A, pulsator \\
\hline W23a & $\mathrm{B} 2 \mathrm{Ia}+\mathrm{OB}$ & $164703.0-455108.0$ & 14.91 & 12.07 & & $\# 6+\# 7$ & & $\mathrm{R}+\mathrm{z}$ & - & A, binary, $\mathrm{H} \alpha$ var., pulsator \\
\hline W24 & O9Iab & $164703.0-455111.0$ & 15.96 & 13.24 & & \#6+\#7 & & $\mathrm{z}$ & - & cand. pulsator \\
\hline W28 & B2Ia & $164704.7-455038.4$ & 14.26 & 11.64 & & $\# 6+\# 7$ & & $\mathrm{R}+\mathrm{Z}$ & & A, $\mathrm{H} \alpha$ var. \\
\hline W29 & O9Ib & $164704.4-455039.8$ & 16.02 & 13.38 & - & & & $\mathrm{R}+\mathrm{z}$ & & \\
\hline W30a & $\mathrm{O}+\mathrm{O}$ & $164705.0-455039.0$ & 15.80 & 13.20 & & $\# 6+\# 7$ & & $\mathrm{R}+\mathrm{z}$ & - & binary, $\mathrm{H} \alpha$ var. \\
\hline W35 & O9Iab & $164704.2-455053.5$ & 16.00 & 13.31 & - & & & $\mathrm{R}+\mathrm{Z}$ & & \\
\hline W41 & O9Iab & $164702.7-455056.9$ & 15.39 & 12.78 & - & & & $\mathrm{R}+\mathrm{z}$ & & \\
\hline W43a & B1Ia & $164603.5-455057.3$ & 15.22 & 12.26 & $\bullet$ & $\# 6+\# 7$ & & $\mathrm{R}+\mathrm{z}$ & $\bullet$ & A, binary, $\mathrm{H} \alpha$ var. \\
\hline W55 & B0Ia & $164659.0-455129.0$ & 15.25 & 12.67 & $\bullet$ & & & & $\bullet$ & $\mathbf{A}$ \\
\hline W60 & BOIab & $164705.0-455151.0$ & 15.96 & 13.28 & & $\# 6+\# 7$ & & $\mathrm{R}+\mathrm{Z}$ & - & $\mathbf{A}$ \\
\hline W61a & B0.5Ia & $164702.3-455141.6$ & 14.62 & 12.01 & & \#6+\#7 & & $\mathrm{R}+\mathrm{z}$ & & A, $\mathrm{H} \alpha$ var. \\
\hline W61b & O9.5Iab & $164702.6-455141.6$ & 16.00 & 13.31 & & $\# 6+\# 7$ & & $\mathrm{z}$ & & \\
\hline W74 & O9.5Iab & $164707.0-455010.0$ & - & - & & & & $\mathrm{R}+\mathrm{z}$ & - & $\mathbf{A}$ \\
\hline W78 & B1Ia & $164702.0-454956.0$ & 14.54 & 12.04 & & & & $R+z$ & - & A, pulsator \\
\hline W238 & B1Iab & $164705.0-455227.0$ & 14.98 & 12.45 & - & & & $\mathrm{R}+\mathrm{z}$ & - & A \\
\hline
\end{tabular}

Notes. Note that W2a was also observed in 2005 March and W61a in 2005 May+July (VLT FLAMES+Medusa). Spectral classifications are from Negueruela et al. (2010). The final column indicates whether a given star is identified as a Periodic or Aperiodic photometric variable, (candidate) binary, $\mathrm{H} \alpha$ variable and/or (candidate) photospheric pulsator (Sect. 3). 